\title{
Acute exercise-induced increases in brain-derived neurotrophic factor in plasma relates to subsequent learning in older adults: A randomized controlled intervention trial
}

Jonna Nilsson $^{1}$ *, Örjan Ekblom ${ }^{2}$, Maria Ekblom² ${ }^{2}$ Benjamin Garzon ${ }^{1}$, Alexander Lebedev ${ }^{1,3}$, Olga Tarassova ${ }^{2}$, Marcus Moberg ${ }^{2} \&$ Martin Lövdén $^{1}$

${ }^{1}$ Aging Research Center, Karolinska Institutet and Stockholm University, Stockholm, Sweden ${ }^{2}$ Swedish School of Sport and Health Sciences, Stockholm, Sweden

${ }^{3}$ Department of Clinical Neuroscience, Karolinska Institutet, Stockholm, Sweden

* Corresponding author

E-mail: jonna.nilsson@ki.se 


\section{Abstract}

Multidomain lifestyle interventions have been identified as a promising strategy to counteract cognitive decline in older age but mechanistic accounts for how such interventions should be designed are lacking. Brain-derived neurotrophic factor is essential for experience-dependent plasticity and increases transiently following physical exercise, suggesting that physical exercise may facilitate subsequent learning by inducing a heightened state of brain plasticity. We therefore hypothesized that cognitive ability in older adults would benefit more from cognitive training when it is immediately preceded as opposed to followed by physical exercise and that the relationship between exercise-induced increases in peripheral BDNF (assessed at pretest) and cognitive training outcomes (change from pretest to posttest) would be greater when cognitive training is preceded by physical exercise. Healthy older adults ( $n=97)$ were randomized to receive either physical exercise immediately before cognitive training, physical exercise immediately after cognitive training, physical exercise only, or cognitive training only, in each training session during a 12-week long intervention period. Consistent with the hypothesis, greater increases of plasma BDNF following physical exercise were associated with greater cognitive training gains on trained task paradigms, but only when such increases immediately preceded cognitive training $(\beta=0.14,95 \%$ CI $[0.04,0.25])$. However, average cognitive training outcome did not significantly differ depending on whether participants received physical exercise before or after cognitive training $(\beta=0.05$, $95 \% \mathrm{CI}[-0.10,0.20])$. The study provides the first empirical support for a time-critical but advantageous role for post-exercise increases in peripheral BDNF for learning in older adults at an interindividual level, with implications for the design of future multidomain interventions. More research will be required to understand the factors that likely interact with and counteract such beneficial effects and to determine whether all older adults could potentially benefit from engaging in physical exercise prior to learning. 


\section{Introduction}

Late-life cognitive impairment and dementia have increasingly serious human, social, and economic burdens, and prevention is a key element to counteract this development (WHO, 2012). Epidemiological studies have found that cognitive performance across the lifespan is associated with various life-style factors, including education (Ritchie \& Tucker-Drob, 2018), occupational complexity (Finkel, Andel, Gatz, \& Pedersen, 2009), and physical activity (Daskalopoulou et al., 2017). Single-domain intervention studies have provided weak support for an advantageous role of cognitive training and physical exercise for cognition in older age, with effects tending to be small and inconsistent (Karbach \& Verhaeghen, 2014; Kelly et al., 2014; Smith et al., 2010; Soveri, Antfolk, Karlsson, Salo, \& Laine, 2017). It has consequently been suggested that multidomain interventions that target several lifestyle factors may be needed for optimal preventative effects for cognitive decline (Ngandu et al., 2015).

Mechanistic accounts for how such multidomain interventions should be designed to optimize preventative effects are, however, largely lacking. Brain-derived neurotrophic factor (BDNF) is a neurotrophin that is essential for neuronal plasticity (Binder \& Scharfman, 2004; Poo, 2001) and increases transiently from physical exercise (Dinoff, Herrmann, Swardfager, \& Lanctôt, 2017; Knaepen, Goekint, Heyman, \& Meeusen, 2010; Szuhany, Bugatti, \& Otto, 2015). BDNF can therefore be expected to be of mechanistic relevance for the interactive effects of physical exercise and cognitive training on cognition. Here we investigate the cognitive effects of a 12-week multidomain intervention comprising cognitive training and physical exercise, in close temporal succession, in healthy older adults, focusing on BDNF as a possible biological mechanism.

Under conditions of physical exercise, the brain is thought to be one of the primary tissues to produce and release BDNF into the blood circulation via the blood-brain barrier (Pan, Banks, Fasold, Bluth, \& Kastin, 1998; Rasmussen et al., 2009). Peripheral BDNF has been found to 
correspond moderately to highly with cortical BDNF in rodents and pigs, which supports the use of peripheral BDNF to gage information about the otherwise inaccessible central BDNF in humans (Karege, Schwald, \& Cisse, 2002; Klein et al., 2011). The vast majority of peripheral BDNF is bound to platelets and only a small fraction is freely circulating in plasma, of which only the latter is immediately bioavailable (Fujimura et al., 2002; Rosenfeld et al., 1995; Walsh \& Tschakovsky, 2018). It is well established that acute exercise is a potent stimulus for transiently increasing peripheral BDNF concentrations in serum as well as in plasma (Dinoff et al., 2017; Knaepen et al., 2010; Szuhany et al., 2015). It has been found that whilst baseline peripheral BDNF levels tend to decrease with age (Erickson et al., 2010; Lommatzsch et al., 2005), acute exercise-related increases do not vary with age (Dinoff et al., 2017; Szuhany et al., 2015), with reliable increases being reported also in older age groups (Håkansson et al., 2017) . Consequently, physical exercise represents a potential route by which BDNF and its presumed neuroplastic consequences can be enhanced for the benefit cognition in old age.

The potentially facilitating effect of physical activity on neuroplastic processes suggests that combining physical and cognitive training interventions may result in an additive effect on cognition (Fujimura et al., 2002; Rosenfeld et al., 1995; Walsh \& Tschakovsky, 2018). A recent meta-analysis of 41 studies investigated the effect of such dual interventions in older adults and concluded that there was no additive effect when comparing the combined intervention to cognitive training alone (Gheysen et al., 2018) . Larger effect sizes were, however, demonstrated for studies that implemented physical exercise and cognitive training simultaneously (e.g. exergames, dance) compared to studies that conducted the interventions in separate sessions, suggesting that the null finding may be due to suboptimal timing of the interventions (Gheysen et al., 2018; Walsh et al., 2016). The temporary nature of the exerciseinduced increase in peripheral BDNF concentrations, with a return to baseline 10-60 min after 
the end of physical activity, suggest that a temporally close succession of cognitive training may be necessary for optimal benefit (Knaepen et al., 2010). Taking such a mechanistic perspective, Walsh et al (2016) proposed that to effectively take advantage of exerciseinduced increases of peripheral BDNF, multidomain interventions should be conducted so that the cognitive engagement immediately follows physical exercise cessation.

The primary aim of the present study was therefore to directly investigate the effect of timing in a combinatory intervention of cognitive training and physical exercise in older adults. To this end, participants were randomized to engage in cognitive training before or after physical exercise, with immediate succession from one intervention to the next in each session, during a 12-week long intervention period. Acute changes in BDNF concentrations, in response to the physical exercise and cognitive training, were assessed at pretest, in serum and in plasma. Given the temporary nature of the exercise-induced BDNF increase and its presumed neuroplastic effects, we hypothesized that cognition in older adults would benefit more from cognitive training when it is preceded as opposed to followed by physical exercise. If supported, the study will provide concrete guidance regarding how physical training should be combined with cognitive training for effectively combatting the increasingly serious challenge of late-life cognitive impairment. On the mechanistic role of BDNF, it was hypothesized that the relationship between exercise-induced increases in peripheral BDNF and cognitive training outcome would be greater when cognitive training is preceded as opposed to followed by physical exercise. If supported, the study will establish a role for exercise-induced increases in BDNF for facilitating learning in older adults. Compared to cognitive training and physical exercise conducted as single-domain interventions, it was furthermore hypothesized that a combined intervention would provide a greater benefit to cognition, independent of order. The hypotheses were pre-registered at the OSF platform 
(Nilsson, J., Ekblom, M., Ekblom, Ö., \& Lövdén, M. (2019, July 1; https://osf.io/wfgr4) and the study will be registered at the ISRCTN registry for clinical trials.

\section{Methodology}

\section{Participants}

Healthy participants between 65 and 75 years were recruited via advertisements in local newspapers. Following an initial screening of study criteria performed via the phone or email, participants were invited to an information meeting. At the meeting, participants were given detailed information about the study and informed consent was obtained from those who were interested in taking part. Study criteria, which were specified to ensure that participants were free of any serious physiological or psychological illness and able to complete the physical as well as the cognitive aspects of the study procedure, were subsequently assessed in full (for complete study criteria, see S1). The Mini Mental State Examination was used to ensure that participants were cognitively intact (scores of $<26$ resulted in exclusion) and a telephone interview conducted by a medical doctor ensured that participants' physical health was sufficient to complete the physical fitness tests. No requirements were imposed in regards to participants' physical fitness or activity level, except that participants had to maintain their previous level of physical activity, in addition to any intervention, during the whole study period. Adherence to this requirement was examined by an accelerometry assessment (see below). After consenting to the study, participants were randomized to one of the four interventions, using age, physical activity level and general cognitive performance as stratifiers. Physical activity level was derived from the added score on two questionnaire items: time spent engaging in activities causing breathlessness in a typical week (i.e. vigorous activity, score range $0-5 ; 0=0 \mathrm{~min}, 1<30 \mathrm{~min}, 2=30-60 \mathrm{~min}, 3=60-90 \mathrm{~min}, 4=90-120 \mathrm{~min}$, 5>120 min) and in everyday physical activities, such as walking and cycling, in a typical week (i.e. moderate physical activity, score range $0-5 ; 0<30 \mathrm{~min}, 1=30-60 \mathrm{~min}, 2=60-90 \mathrm{~min}$, 
$3=90-150 \mathrm{~min}, 4=150-300 \mathrm{~min}, 5>300 \mathrm{~min}$ ). An objective assessment of physical activity pattern (accelerometry) and of cardiovascular fitness (maximal treadmill ergometer test) were conducted after randomization. General cognitive ability was derived from performance on a progressive matrix reasoning test performed before randomization (score range $0-18$ ). The study was approved by the ethical review board in Stockholm (Regionala Etikprövningsnämnden, Stockholm, case number 2017/1115-31/4) and conducted in accordance with the Declaration of Helsinki.

\section{Design and procedure}

The study procedure consisted of a pretest phase, an intervention phase and a posttest phase, with random assignment to intervention (Figure 1). The intervention phase lasted for 12 weeks with training visits occurring every second week day (two or three times per week on alternating weeks), resulting in a maximum of 30 training visits during the intervention period. At each training visit, participants received the intervention allocated at randomization. The physical exercise $(\mathrm{PE})+\operatorname{cognition}(\mathrm{COG})$ and the $\mathrm{COG}+\mathrm{PE}$ groups engaged in both physical exercise and cognitive training at each training visit and differed only in the order in which the training types were completed. The PE+COG group completed the physical exercise regime first and then immediately the cognitive training whilst the $\mathrm{COG}+\mathrm{PE}$ group received the training types in the reverse order. The COG group engaged only in cognitive training and the PE group engaged only in physical exercise at each training visit. All training visits were conducted in a group setting, with a maximum group size of six. Training visits were always scheduled between 08:00 and 12:30 and training time was kept constant for each participant throughout the intervention period. Training time was also counterbalanced across the intervention types so that each training session started on average at the same time in the morning for the four intervention groups. All training sessions started with 15 minutes of seated rest, independent of intervention. The pretest and the posttest phase 
were completed in the week before and after the intervention and included an extensive cognitive assessment, physical fitness tests, accelerometer measurements, and assessment of acute changes of peripheral BDNF in response to the allocated intervention type (Figure 1).

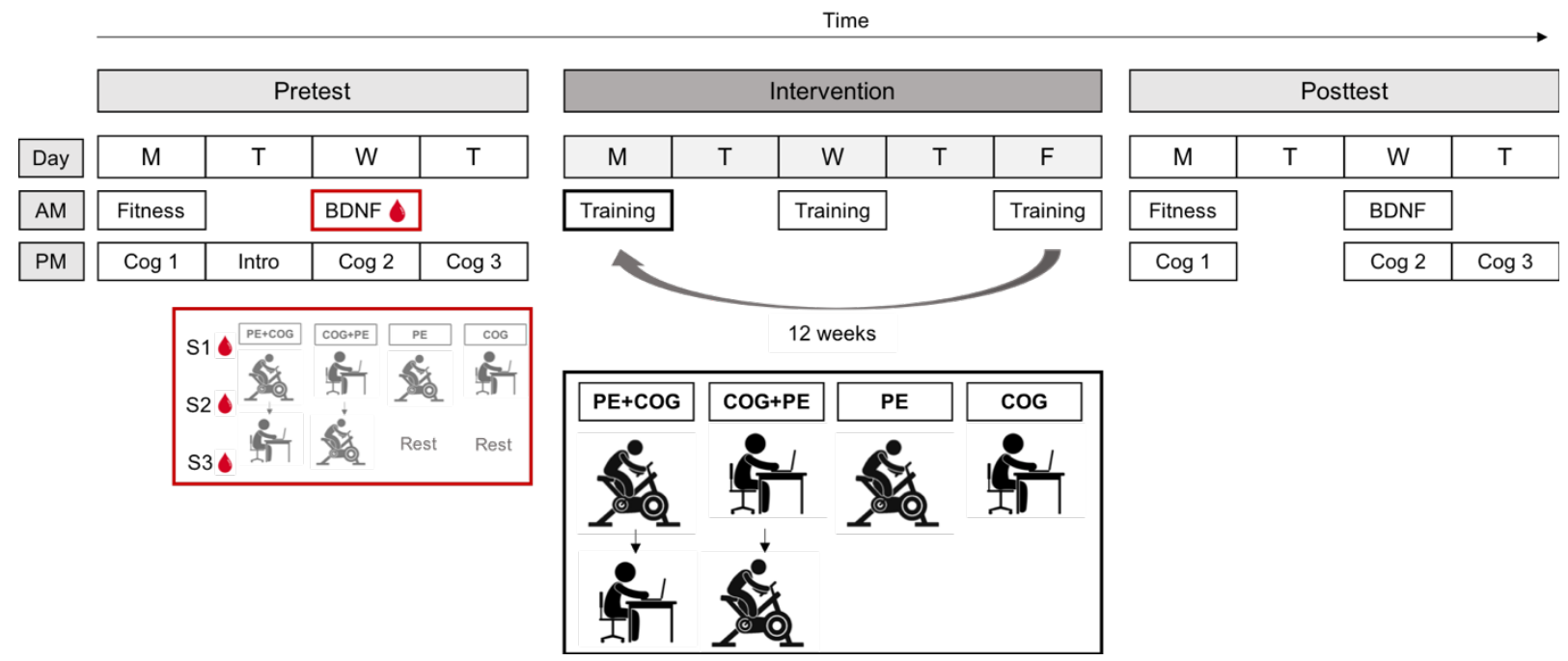

Figure 1. Schematic of the study procedure. The pretest and posttest phases included physical fitness tests (Fitness) and an assessment of acute changes in BDNF concentrations (BDNF), both performed in the morning and at least 48 hours apart, as well as an extensive cognitive assessment completed over three afternoon sessions (Cog 1-3). The pretest phase additionally included an introduction to the allocated training intervention (Intro). At the BDNF assessment, the allocated intervention was performed and three blood samples were obtained: before (S1) and after the first intervention (S2) and after the second intervention or rest (S3; see red inset). During the intervention phase, training sessions took place every second weekday, in the morning ( 2 or 3 days per week on alternating weeks; 3-day week represented in figure), according to the allocated intervention: physical exercise followed by cognitive training $(\mathrm{PE}+\mathrm{COG})$, cognitive training followed by physical exercise (COG+PE), physical exercise only (PE) and cognitive training only (COG). The cognitive training involved 35 minutes of adaptive working memory training and the physical exercise involved 35 minutes of aerobic exercise on a stationary bike at $65-75 \%$ of $\mathrm{VO}_{2}$-max. The accelerometer assessment was performed in the week before the intervention and in the last intervention week (not depicted). Note that a BDNF assessment was also conducted at posttest but was not of interest in the present study.

Cognitive training. The computerized cognitive training program targeted the working memory construct of updating. In each session, participants trained on an n-back task and a running span task, both of which require continuous maintenance and updating of mental 
representations ( $\mathrm{S} 2$ for detailed task descriptions). Two blocks of n-back (6 runs/block) were alternated with two blocks of running span ( 2 runs/block) in each session, alternating between which task that was presented first from session to session. After each run, performance was evaluated against a fixed criterion and if the criterion was met, progression to the subsequent difficulty level with a greater working memory load followed. As such, the difficulty of the task increased adaptively as participants improved in their ability to perform the tasks. Each task had a total of 21 levels and participants who reached the highest level repeated this level for the rest of the intervention period. Participants received figural feedback on their performance after each run and additionally on their training progress every second week via a print-out of level progression across the training visits completed so far. Improvements in processing efficiency over strategy-based processes were promoted by the inclusion of the two updating tasks (n-back, running span) as well as four different stimuli sets for each task type (S2 for detailed task descriptions). The test leader was available for support and guidance throughout the session.

After each cognitive training session, participants completed an additional n-back task, which was identical across the study period (i.e. non-adaptive) to allow for an assessment of learning as a result of the cognitive training ( $\max$ score $=24 ; \mathrm{S} 3$ for detailed description of the daily $n$ back task). Each cognitive training session lasted for approximately 35 minutes, of which 30 minutes were spent on the adaptive training tasks and 5 minutes on the non-adaptive n-back task. Thirty cognitive training sessions were completed during the intervention phase and two were completed as part of the BDNF assessment at pretest and posttest, resulting in a maximum number of 32 cognitive training sessions. To ensure that participants who had been allocated cognitive training were familiarized with the training program prior to the BDNF assessment and the start of the intervention period, an introduction to the training program was given during the pretest phase. In addition to receiving information about the training 
tasks, the procedure for level progression and the types of feedback, participants were given the opportunity to try the training tasks at different difficulty levels and with different stimuli sets.

Physical exercise. Each physical exercise session lasted for approximately 35 minutes, of which 5 minutes were spent warming up and 30 minutes on engaging in moderate aerobic exercise at a heart rate corresponding to $65-75 \%$ of participants' individual maximum heart rate (HR), as indicated by the fitness assessment conducted at pretest. More specifically, the intensity was systematically varied in six 5-minute intervals by instructing participants to maintain a heart rate that corresponded to $65 \%, 70 \%, 75 \%, 70 \%, 75 \%$, and $65 \%$ of their individual HR during the respective intervals. The exception to this routine were the three first exercise sessions, which acted as a warm-in sessions and implemented alternating 5minute intervals with a heart rate that corresponding to $65 \%$ and $70 \%$ of the individual $\mathrm{HR}$. Heart rate was measured using a chest-strap heart rate monitor Polar monitor (m400, Polar Electro Oy, Kempele, Finland) and was displayed to allow for self-monitoring based on individualized HR cards attached to the exercise bike, which stated the appropriate intensity intervals for each participant. Towards the end of each exercise interval, the heart rate and participants' rated perceived exertion (RPE; Borg., 1970) were monitored by the test leader. If participants had trouble reaching or maintaining their heart rate, additional guidance on how to appropriately change the resistance or the speed of pedaling was provided by the test leader. If participants' RPE was outside the range of 13 ("somewhat hard") to 15 ("hard"), participants were instructed to increase or reduce the intensity accordingly. As such, the instructed intensity was intended to allow for possible improvements infitness. Videos of cycling rides in the Stockholm area were displayed on a large screen to make the exercise sessions more motivating. The test leader was available for support and guidance throughout the session. Only water was allowed during the exercise sessions. To ensure that participants 
who had been allocated physical exercise were familiarized with the nature of the training sessions, an introduction was given prior to the BDNF assessment and the start of the intervention period. Participants were instructed on how to appropriately adjust the bike and how to maintain the appropriate exercise intensity by varying resistance and cadence, in accordance with the individual heart rate range and by feedback from the test leader on perceived level of exhaustion.

Combined physical exercise and cognitive training. Participants in the $\mathrm{PE}+\mathrm{COG}$ and the $\mathrm{COG}+\mathrm{PE}$ groups received identical cognitive and physical training sessions as those in the COG and PE groups. In both combined groups, bikes were adjusted and heart rate monitors fitted prior to the start of the session and participants were instructed to move as quickly as possible between the exercise bike and the computer. In the $\mathrm{PE}+\mathrm{COG}$ group, participants were allowed a brief moment to have some water and to put on a sweater before sitting down at the computer, at which a 1-minute countdown passed before the cognitive training started. In the $\mathrm{COG}+\mathrm{PE}$ group, participants moved to the exercise bikes and started the warm-up as soon as the cognitive training session had ended.

Cognitive assessment. The cognitive assessments at pretest and posttest consisted of a total of 19 tests, of which 18 were intended for measuring eight cognitive constructs of interests (S4 for detailed task descriptions). The cognitive constructs were selected to vary in similarity to the training tasks included in the cognitive training protocol: trained working memory tasks (trained stimuli), trained working memory tasks (untrained stimuli), untrained working memory tasks (updating and switching) and untrained cognitive domains (episodic memory, processing speed, spatial reasoning and verbal reasoning). The spatial reasoning and verbal reasoning domains were assessed using three different tests and the remaining domains were assessed using two tests. The trained working memory tasks (trained stimuli) and (untrained stimuli) both used the same n-back and running span tasks as in the cognitive training 
intervention, but differed in that the former additionally used the same stimuli as in the intervention whilst the latter used novel stimuli. All cognitive tests were computerized with the exception of one spatial reasoning measure (adapted Raven's progressive matrices), which was completed in pen-and-paper format. Instructions were provided orally by the test leader in combination with visual depictions of the task procedure on participants' individual computer screens. For each task, participants completed practice trials and had an opportunity to ask questions prior to proceeding with the actual task. No feedback on performance was provided. The cognitive tests were completed over three sessions in the week before and the week after the intervention in groups of maximum eight participants, keeping task order the same. All cognitive assessment sessions were scheduled in the afternoon and were kept the same at pretest and posttest (14:00-16:30).

Fitness assessment. Participant's cardiovascular fitness was assessed at pretest and posttest, using a maximal treadmill ergometer test, preceded by a submaximal cycle ergometer test. A submaximal test was completed to ensure a measurement of cardiorespiratory fitness and a calculation of exercise intensity also for those participants who are not able to complete the maximal treadmill test. The fitness assessment was completed in the first half of the day $(08: 30-12: 30)$ in the week before and after the intervention, with all participants being tested individually and at the same time at pretest and posttest. A minimum of 48 hours passed between the fitness assessment and the BDNF assessment. For both fitness tests, heart rate (HR) was measured using a heart rate sensor on the chest and a Polar monitor (m400, Polar Electro Oy, Kempele, Finland).

The submaximal cycle ergometer test was performed on a cycle ergometer (model 828E, Monark, Varberg, Sweden) prior to the maximal test. The Ekblom-Bak test (EB-test) protocol was used (Björkman et al., 2016) including two steps: 4 minutes cycling at a standard work rate (resistance of $0.5 \mathrm{kp}$, cadence of 60 revolutions per minute) and $4-5$ minutes cycling at a 
higher work rate $(50-70 \%$ of maximal capacity, determined individually by a steady state HR of 110-140 bpm and RPE of around but not above 16). Mean HR was calculated from the last minute on each work rate. $\mathrm{VO}_{2}$ max was estimated using sex-specific prediction equations based on data from EB-test, based on HR at standard work rate, a factor for the higher work rate, change in HR between the standard work rate and the higher work rate, age and sex (Björkman et al., 2016). $\mathrm{VO}_{2}$ max results from the submaximal test were expressed in relative $(\mathrm{mL} / \mathrm{kg} / \mathrm{min})$ units.

Maximum rate of oxygen consumption $\left(\mathrm{VO}_{2}\right)$ max and maximal heart rate reserve were measured directly from the maximal treadmill ergometer test. Before the initiation of the maximal treadmill ergometer test, a 5-10 minutes warm-up was performed to give a participant an opportunity to get familiar with the equipment. The maximal test started with an initial treadmill incline of 1 degree at a comfortable speed (around RPE 12-13) and continued with increases of incline and/or speed every minute until volitional exhaustion. Participants wore a harness attached to the ceiling to protect from falls and to increase confidence and thereby maximize the chances of reaching volitional exhaustion. $\mathrm{VO}_{2} \max$ was measured using a computerized metabolic system, using the breath-by-breath mode (Jaeger Oxycon Pro, Hoechberg, Germany) . Similar to previous studies (Björkman, EkblomBak, Ekblom, \& Ekblom, 2016; Ekblom-Bak, Björkman, Hellenius, \& Ekblom, 2014), the VO2max measurement was accepted if a minimum of three out of five following criteria were achieved: a) $\mathrm{VO}_{2}$ was leveling off despite an increase in speed or decline, b) RPE reached above $16, \mathrm{c})$ a respiratory exchange ratio reached value greater than $1.1, \mathrm{~d}$ ) maximal HR within \pm 15 beats per minute from age-predicted maximal HR and e) a work with time above 6 minutes was performed. The highest 30 seconds of registered values of $\mathrm{VO}_{2}$ and heart rate were referred to as $\mathrm{VO}_{2}$ max and maximum $\mathrm{HR}$, respectively. $\mathrm{VO}_{2}$ max results were expressed in relative $(\mathrm{mL} / \mathrm{kg} / \mathrm{min})$ units and maximum $\mathrm{HR}$ in beats per minute (bpm). 
BDNF assessment. The blood sampling protocol was designed to measure acute changes in BDNF concentrations in response to the different interventions. The assessment was performed before and after the intervention period but since the posttest measures were not of relevance for the posed hypotheses they are not considered here. The BDNF assessment for each participant individually and was completed at least 48 hours after the fitness assessment. In order to make the BDNF assessment representative of a typical training session, the session was always scheduled for the first half of the day (08:00-12:30), at the same or similar time of day as the individual participant's training sessions during the intervention.

At pretest, upon arrival, a peripheral intravenous catheter was inserted into the antecubical vein to facilitate timely blood sampling. After 15 minutes of seated rest, the first blood sample was drawn. Participants subsequently commenced with cognitive training or physical exercise, depending on the intervention allocated at randomization, after which the second blood sample was collected. Participants then engaged in cognitive training, physical exercise or seated rest, again depending on intervention, after which the third sample was drawn. As such, three blood samples were taken at pretest from all participants. To avoid contamination between blood samples, the catheter was flushed with saline solution between three blood samples. For each blood sample, a total of $10 \mathrm{~mL}$ was collected into (a) heparinized containers (plasma), and (b) sterile separator tubes (serum). After blood collection, the blood sampled in the heparinized container were spun in an Eppendorf 5415R centrifuge (Eppendorf Canada Ltd, Ontario, Canada) at $4{ }^{\circ} \mathrm{C}$ for $3 \mathrm{~min}$ at $10000 \mathrm{~g}$ (rcf) to separate the plasma, which was placed in a new container and frozen at $-80{ }^{\circ} \mathrm{C}$ until analysis. The blood sampled in the serum tubes additive-free container was left for 30 minutes to clot after which it was spun in a Sigma 3K12 centrifuge (Sigma Laborzentrifugen GmbH, Osterode am Harz, Germany) at 8 ${ }^{\circ} \mathrm{C}$ for $10 \mathrm{~min}$ at $2500 \mathrm{rpm}$ to separate the serum, which was then placed in a new container and frozen at $-80{ }^{\circ} \mathrm{C}$ until analysis. BDNF concentrations were quantified using an enzyme- 
linked immunosorbant assay according to the manufacturer's instructions (Human BDNF Quantikine Immunoassay, DBD00, R \& D Systems). All samples were run in duplicate and each data point is the mean value of the duplicate. Due to the high concentration of BDNF in serum these samples were diluted 20x in assay diluent in order to fit the assay standard curve. Accelerometry assessment. The accelerometry assessment was performed to assess potential reactive or compensatory changes in participants' movement outside the intervention, in response to study participation, and to ensure that such changes did not differ between the intervention groups. To this end, accelerometers were worn for 7 days before the first study visit, reflecting participants' usual pattern of movement, and for the last 7 days of the intervention, reflecting potential changes to the pattern of movement due to intervention engagement. Since the accelerometers primarily capture locomotion activity and underestimate non-ambulatory activities, such as cycling, the accelerometer assessment can be assumed to reflect physical activity primarily outside the physical exercise intervention. Accelerometers were lightweight and worn in an elastic elastic band on the hip during the day and on the non-dominant wrist during the night (Actigraph GT3X+, Actigraph LCC; Pensacola, FL, USA). The accelerometer data were recorded as raw data (sample rate set to $30 \mathrm{~Hz}$ ) from all three axes, which were combined into a resulting vector. Using the ActiLife v 6 software, raw data were transformed into the arbitrary unit "counts per minute", and extracted as a mean over a 60 seconds epoch using a low frequency extension filter. A minimum of 600 minutes of valid daily wear time for at least 4 days was required to be included in the analyses (Trost, McIver, \& Pate, 2005). Using standard definitions, sedentary behaviours was defined as $<200$ counts per minute (cpm) and moderate-to-vigorous physical activity as cpm $\geq 2690$ (Sasaki, John, \& Freedson, 2011). Percentage time spent between 06:00 and 23:00 in the different intensities constituted measures of interest.

\section{Statistical analysis}


Cognitive composites. Cognitive composites were created by taking the unit-weighted average of the two or three relevant measures at pretest and posttest (S4). Composite scores (x) were subsequently standardized by pretest performance ((x-mean pretest $\left.) / \mathrm{sd}_{\text {pretest }}\right)$ and converted into $\mathrm{T}$ scores $\left(\operatorname{mean}_{\text {pretest }}=50, \mathrm{sd}_{\text {pretest }}=10\right)$. All cognitive composite scores were approximately normally distributed (skewness $<3.0$, kurtosis $<10.0$ ). Descriptive statistics for all cognitive composites at pretest and posttest are available for each group in the supplementary information (S5).

BDNF. BDNF concentrations in serum were normally distributed at all three sampling timepoints (skewness $<3.0$, kurtosis $<10.0$ ), with no extreme outliers detected (outlier labelling rule, $\mathrm{IQR}=3.0$ ). In contrast, $\mathrm{BDNF}$ concentrations in plasma were severely negatively skewed at all sampling timepoints (skewness $>3.0$, kurtosis $>10.0$ ). Natural log-transformations were therefore performed, which were successful in making the distributions normal at all sampling timepoints, with no extreme outliers being subsequently detected. For analyses in which acute BDNF change following physical exercise was used as a predictor of cognitive training outcome, the concentration measured just before exercise was subtracted from the concentration measured immediately after. For the PE and PE+COG groups (PE first intervention), this meant that sample 1 was subtracted from sample 2 , and for the COG+PE group (PE second intervention), that sample 2 was subtracted from sample 3 (Figure 1). Fitness. Only direct measurements of $\mathrm{VO}_{2}$ max were included in analyses of physical fitness. Exercise intensity intervals were based on the $\mathrm{VO}_{2}$ max measurements from the maximal test when available $(\mathrm{n}=94)$ and otherwise on $\mathrm{VO}_{2}$ max estimates from the submaximal test $(\mathrm{n}=3)$. Linear mixed-effect models. Linear mixed-effects models (LMM) were fitted using the lme4 package (version 1.1-18-1, (Bates, Maechler, Bolker, \& Walker, 2015)) in the R programming environment (version 3.5.1, (R Core Team, 2017)) employing restricted maximum likelihood (REML) to estimate the parameters. Inferential analyses were performed using the lmerTest 
package (version 3.1-0, (Kuznetsova, Brockhoff, \& Christensen, 2017)) using the Satterthwaite's approximation to estimate denominator degrees of freedom for $F$ statistic and to obtain p-values (type III analysis of variance). All possible interactions between fixed factors were included in all models. For analyses concerned with change in cognition from pretest to posttest, intercept for subject was entered as a random factor. In analyses on change in performance across all cognitive training visits, intercept and linear slope were entered as random factors. We corrected for testing multiple models based on the number of models used to test a single hypothesis, using Bonferroni-corrected $\alpha$-levels. Post-hoc tests were performed where appropriate to follow up on significant interactions to aid interpretation. In the case of mean differences, estimated marginal means were contrasted using the emmeans package (version 4.1), employing the Tukey method to adjust for multiple comparisons and Satterthwaite's approximation for determining degrees of freedom. In the case of differential relationships, relevant zero-order correlations were computed and compared using Fisher's r to $\mathrm{z}$ transformation. No other correction of the $\alpha$-level was applied for post-hoc follow-up analyses. Visual inspection of residual plots did not reveal any obvious deviations from homoscedasticity or normality. The details of the models are presented in the context of the hypotheses in the Results section.

\section{Results}

\section{Descriptive results}

In total, 139 participants were randomized, of which 97 completed the study and were included in the analyses (Figure 2). The majority of drop-outs occurred before the first study visit, with an equivalent number of drop-outs $(n=3-5)$ for the four different intervention groups after study start. The effects of dropout were small with no evident differential effects in the four intervention groups, with standardized mean differences (SMD) between the randomized and final sample in intervention groups ranging from 0.06 to 0.15 for age, from 
for self-reported time spent engaging in vigorous, moderate and sedentary activity, respectively (for complete drop-out analysis, see S6).

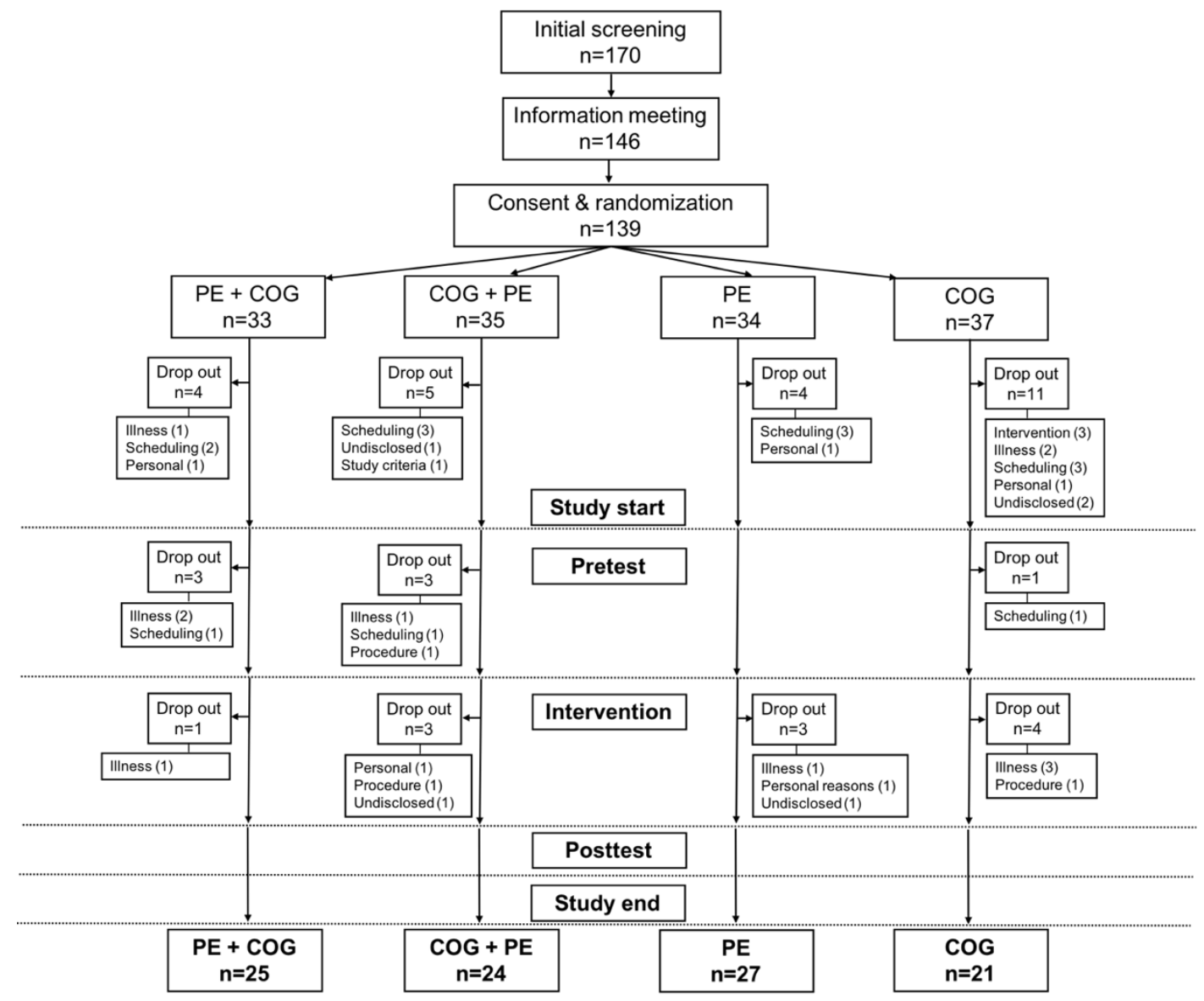

Figure 2. Recruitment diagram. Number of subjects initially screened, attending the information meeting, consenting to, being randomized to and completing each of the four intervention programs $(\mathrm{PE}+\mathrm{COG}, \mathrm{COG}+\mathrm{PE}$, PE, COG). Number of drop-outs is specified for each phase of the study procedure and reasons discontinuing the study are specified in brief. For drop-out analysis, see S6. Illness=illness, injury or health reasons.

Scheduling=inability to adhere to the study schedule. Personal= personal reasons. Undisclosed $=$ reason not specified. Study criteria=one or more study criteria no longer fulfilled. Intervention=dissatisfied with allocated intervention. Procedure=dissatisfied with one or more aspects of the study procedure.

For the final sample, participants in the four intervention groups were comparable in regards to basic demographic variables (age, sex, education), physiological measures (BMI, $\mathrm{VO}_{2}$ max, maximum HR), physical activity level (time spent engaging in sedentary and moderate- 
vigorous activity, as derived from the accelerometry assessment) and general cognitive ability (MMSE, Reasoning) at pretest (Table 1).

Table 1. Demographic, physiological and cognitive information at pretest for the final the study sample. $\mathrm{VO}_{2}$ max here represents direct measurements derived from maximal treadmill test, which was available for 94 participants. Maximal heart rate (HR) was derived from the maximal treadmill test where available $(n=93)$ and otherwise from the submaximal ergometer test $(\mathrm{n}=4)$. Physical activity measures were derived from the accelerometry assessment, considering time between 06:00 and 23:00. Education level: 1=elementary school, 2=high school, 3=university. Mini Mental State Exam: maximum score 30, score. $\mathrm{BMI}=$ body max index, $\mathrm{VO}_{2}$ $\max =$ maximum rate of oxygen consumption, $\mathrm{MMSE}=$ Mini mental state exam (max score $30,<26$ excluded), Reasoning=progressive matrix reasoning ( $\max$ score 18), $\mathrm{PE}=$ physical exercise, $\mathrm{COG}=$ cognitive training.

\begin{tabular}{|c|c|c|c|c|c|c|c|c|c|c|c|c|c|}
\hline & & \multicolumn{3}{|c|}{ COG } & \multicolumn{3}{|c|}{ PE } & \multicolumn{3}{|c|}{ COG+PE } & \multicolumn{3}{|c|}{ PE+COG } \\
\hline & & $n$ & mean & $s d$ & $n$ & mean & $s d$ & $n$ & mean & $s d$ & $n$ & mean & $s d$ \\
\hline \multirow[t]{3}{*}{ Demographics } & Age (years) & 21 & 70,95 & 3,04 & 27 & 70,30 & 3,00 & 24 & 70,29 & 3,11 & 25 & 70,28 & 2,70 \\
\hline & Education (level) & 21 & 2,71 & 0,64 & 27 & 2,52 & 0,80 & 23 & 2,70 & 0,56 & 25 & 2,68 & 0,56 \\
\hline & Sex (females/males & 21 & $60 / 40$ & - & 27 & $56 / 44$ & - & 24 & $48 / 52$ & - & 25 & $52 / 48$ & - \\
\hline \multirow[t]{3}{*}{ Physiology } & BMI (kg/m2) & 21 & 25,21 & 3,45 & 26 & 26,23 & 3,17 & 22 & 26,98 & 4,34 & 25 & 25,05 & 3,69 \\
\hline & VO2 $\max (\mathrm{mL} / \mathrm{kg} / \mathrm{min})$ & 21 & 31,01 & 5,00 & 25 & 30,71 & 5,06 & 23 & 31,00 & 6,03 & 24 & 32,99 & 5,82 \\
\hline & Max HR (beats/min) & 21 & 167,60 & 10,03 & 27 & 161,50 & 15,39 & 24 & 164,90 & 11,16 & 25 & 162,20 & 12,42 \\
\hline \multirow[t]{2}{*}{ Physical activity } & Sedentary activity (\% time) & 20 & 57,00 & 10,00 & 27 & 55,00 & 9,00 & 23 & 56,00 & 8,00 & 25 & 56,00 & 6,00 \\
\hline & Moderate-vigorous activity (\% time) & 20 & 6,00 & 3,00 & 27 & 6,00 & 2,00 & 23 & 6,00 & 2,00 & 25 & 7,00 & 3,00 \\
\hline \multirow[t]{2}{*}{ Cognition } & MMSE (score) & 21 & 29,24 & 0,83 & 27 & 29,52 & 0,75 & 24 & 29,33 & 1,05 & 25 & 29,32 & 1,15 \\
\hline & Reasoning (score) & 21 & 5,86 & 2,95 & 27 & 6,04 & 2,77 & 24 & 6,50 & 3,07 & 25 & 6,16 & 2,48 \\
\hline
\end{tabular}

Attendance. The intervention groups did not differ in regard to the number of attended training sessions, $F(1,95)=0.256, p=0.614$, with the $\mathrm{PE}+\mathrm{COG}$ group attending 24.48 sessions $(\mathrm{SD}=2.02)$, the $\mathrm{COG}+\mathrm{PE}$ group 25.17 sessions $(\mathrm{SD}=2.46)$, the $\mathrm{COG}$ group 25.05 sessions $(\mathrm{SD}=2,50)$ and the $\mathrm{PE}$ group 24.63 sessions $(\mathrm{SD}=2.71)$. In the groups that received both cognitive training and physical exercise (COG+PE, $\mathrm{PE}+\mathrm{COG})$, occasionally only one of the intervention types could be completed, predominantly due to residual cold symptoms in the case of physical exercise and to technical problems in the case of cognitive training. Considering only training sessions completed in full, participants in the $\mathrm{PE}+\mathrm{COG}$ group completed 23.48 sessions $(\mathrm{SD}=4.30)$ and participants in the $\mathrm{COG}+\mathrm{PE}$ group completed 24.58 $(\mathrm{SD}=2.34)$, with no significant difference between the four intervention groups, $F(1,95)=2.881, p=0.093$. 
Accelerometry. Accelerometer data was available for 95 participants for the week before study start $(\mathrm{COG}=20, \mathrm{PE}=27, \mathrm{COG}+\mathrm{PE}=23, \mathrm{PE}+\mathrm{COG}=25)$ and for 90 participants for the last week of the intervention $(\mathrm{COG}=17, \mathrm{PE}=26, \mathrm{COG}+\mathrm{PE}=23, \mathrm{PE}+\mathrm{COG}=24)$. Since the accelerometers primarily capture locomotion activity and underestimate non-ambulatory activities, such as cycling, the accelerometer assessment reflected physical activity primarily outside the physical exercise intervention. To assess potential changes in patterns of physical activity pattern as a result of study participation, linear mixed models were applied, with group (COG, PE, PE+COG, COG+PE) and time (week before intervention, last week of intervention) as fixed factors and subject intercept as random factor, focusing on daytime physical activity (06:00-23:00). Participants engaged in more prolonged sedentary bouts in the last week of the intervention $(\mathrm{M}=58.3 \%, \mathrm{SD}=8.10)$ compared to the week before study start $(\mathrm{M}=55.9 \%, \mathrm{SD}=8.47), F(1,91.38)=6.069, p=0.0156$, but importantly the intervention groups did not differ in overall sedentary behavior in a statistically significant way, $F(1$, $175.12)=0.1856, p=0.667$, or in change of sedentary behavior, $F(1,90.95)=1.168, p=0.283$. Participants spent $6.40 \%(\mathrm{SD}=2.50)$ of their time engaged in moderate-to-vigorous physical activity prior to study start and this did not change in a statistically significant way as a result of the intervention, $F(1,90.38)=0.970, p=0.327$. The intervention groups did not also not significantly differ in overall, $F(1,158.90)=0.027, p=0.869$, or in change of moderate-tovigorous activity, $F(1,90.80)=1.167, p=0.283$. As such, the different interventions do not appear to have resulted in any pronounced differential compensatory changes in physical activity patterns outside of the intervention itself.

$B D N F$ assessment. All three blood samples were successfully drawn from all participants at pretest. However, four serum samples were not handled according to protocol were therefore excluded from analyses (sample 1 for one participant in the COG+PE group, samples 1-3 for one participant in the COG group and one in the PE group), resulting in available BDNF 
concentrations in serum for 94 participants for sample 1 and for 95 participants for sample 2 and 3. Concentrations in plasma were available for all 97 participants for all three samples. Mean resting BDNF concentration at pretest was $24673 \mathrm{pg} / \mathrm{mL}$ in serum $(S D=5600)$, ranging from 11598 to 46013 . Median resting concentration was $141 \mathrm{pg} / \mathrm{mL}$ in plasma (IQR=193), ranging from 28 to $6820 \mathrm{pg} / \mathrm{mL}$. The average intensity of physical exercise during the BDNF assessment was $72.4 \%$ of the individual maximum heart rate, with no significant difference between groups (PE, PE+COG, COG+PE), $F(1,73)=0.308, p=0.58$.

\section{Hypothesis testing}

Hypothesis testing was performed in accordance with the pre-registration (osf.io/9m6aj) and results are presented below (pre-registration numbering of hypotheses in brackets), after which exploratory analyses are presented.

The acute change in peripheral BDNF levels in older adults will be greater immediately following physical exercise compared to immediately following working memory training (H5)

This hypothesis concerned blood samples taken before and after the first intervention type at pretest (ordered factor: sample $1<$ sample 2). The groups were coded according to the first intervention: $\mathrm{PE}$ and $\mathrm{PE}+\mathrm{COG}$ were combined $\left(1^{\text {st }}\right.$ intervention $\left.=\mathrm{PE}\right)$, and, $\mathrm{COG}$ and $\mathrm{COG}+\mathrm{PE}$ were combined $\left(1^{\text {st }}\right.$ intervention $\left.=\mathrm{COG}\right)$. BDNF concentrations in serum and plasma were evaluated in separate models $\left(\alpha_{\text {corr }}=0.025\right)$.

For serum, there was a significant interaction between intervention and time, $F(1,92.3)=26.6$, $p<0.0001$, and a significant effect of time, $F(1,92.3)=50.65, \mathrm{p}<0.0001$. Post-hoc pairwise comparisons revealed that BDNF concentrations in serum increased significantly from sample 1 to sample 2 following PE, $t(92.2)=-9.07, p<0.0001$, but not following COG, $\mathrm{t}(92.4)=-1.331$, $p=0.1866$, in support of the hypothesis. For plasma, there was no interaction between intervention and time, $F(1,94.8)=0.0057, p<0.9398$, but a significant effect of time, $F(1$, 
$94.8)=117.2872, p<0.0001$, demonstrating a reliable increase from sample 1 to sample 2 across groups, $\mathrm{t}(94.8)=-10.801, \mathrm{p}<0.0001$. The acute mean change in BDNF concentrations are visualized for all intervention groups in Figure 3.

In descriptive terms, following physical exercise, mean serum concentrations increased from $23964 \mathrm{pg} / \mathrm{mL}(S D=4979)$ to $28244 \mathrm{pg} / \mathrm{mL}(S D=6365)$, reflecting an average $18 \%$ change, whilst non-transformed median plasma concentrations increased from $192 \mathrm{pg} / \mathrm{mL}(I Q R=352)$ to $802 \mathrm{pg} / \mathrm{mL}(I Q R=898)$, reflecting an increase of over $300 \%$. Following cognitive training, mean serum concentrations demonstrated virtually no average change from $25011 \mathrm{pg} / \mathrm{mL}$ ( $S D=4958)$ to $25238 \mathrm{pg} / \mathrm{mL}(S D=4510)$, whilst non-transformed median plasma concentrations increased from $129 \mathrm{pg} / \mathrm{mL}(I Q R=156)$ to $388 \mathrm{pg} / \mathrm{mL}(I Q R=510)$, reflecting a $200 \%$ increase. All relevant descriptive statistics are available in S7. 
A)

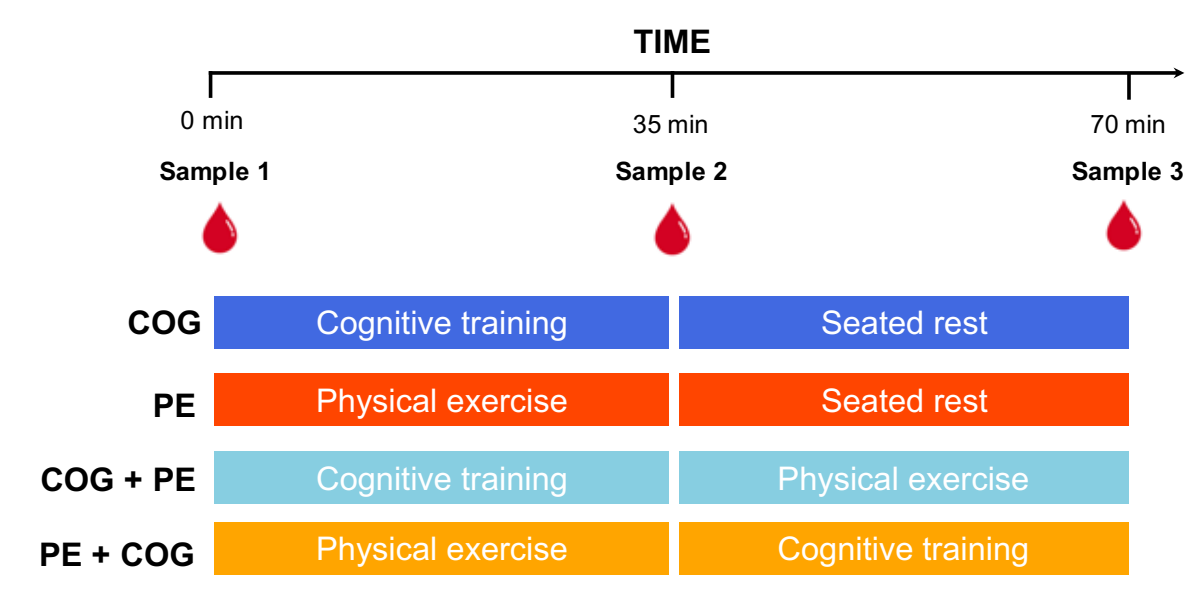

B)

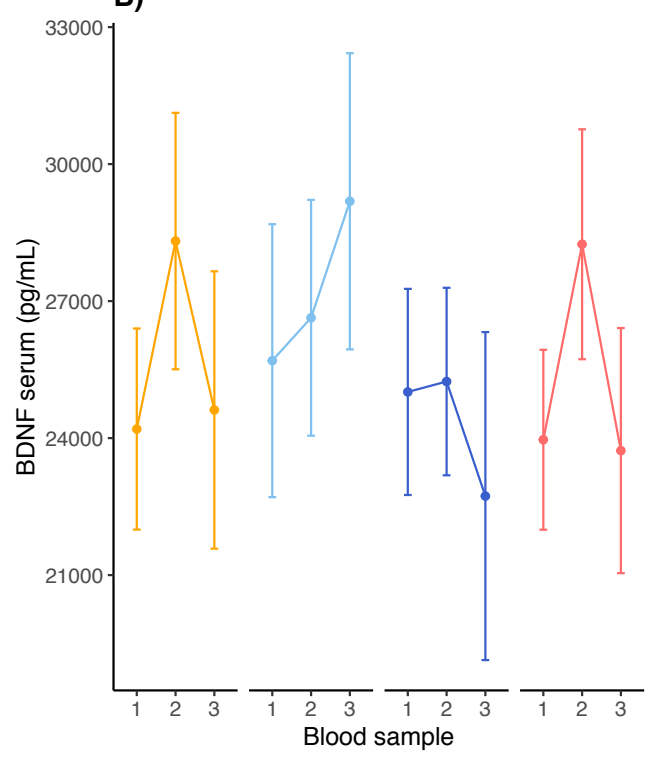

C)

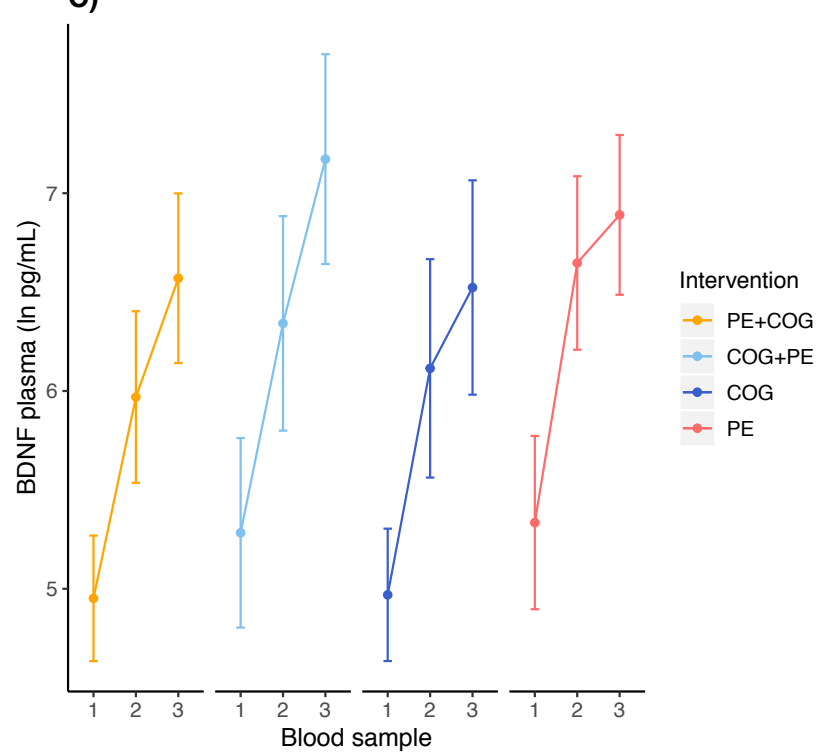

Figure 3. Overview of the blood sampling protocol that was used at pretest to assess acute changes in BDNF concentration in response to the four interventions: COG (dark blue), PE (red), COG+PE (light blue) and $\mathrm{PE}+\mathrm{COG}$ (orange; A). Acute changes in BDNF concentrations in response to the interventions at pretest, in serum (B) and in plasma natural log transformed (C). BDNF concentrations in serum increase in response to physical exercise (sample 1 to 2 in PE and PE+COG groups; sample 2 to 3 in COG+PE group) but not in response to cognitive training (sample 1 to 2 in $\mathrm{COG}$ and $\mathrm{COG}+\mathrm{PE}$; sample 2 to 3 in $\mathrm{PE}+\mathrm{COG}$ group). $\mathrm{BDNF}$ concentrations in plasma increase independent of intervention. Note that the hypothesis testing involved specific group comparisons and merging of groups. 
The acute changes in peripheral BDNF levels in older adults following physical exercise and cognitive training will remain after 30 minutes (H6)

This hypothesis concerned blood samples taken after the first intervention and after 30 minutes seated rest at pretest (ordered factor: sample $2<$ sample 3 ). The analyses were restricted to the groups with only one intervention (PE, COG). BDNF concentrations in serum and plasma were evaluated in separate models $\left(\alpha_{\text {corr }}=0.025\right)$.

For serum, there was no statistically significant interaction between intervention (COG, PE) and time (sample 2, sample 3 ), $F(1,44)=1.8011, \mathrm{p}=0.1865$, but a significant effect of time, $\mathrm{F}(1,44)=22.0243, \mathrm{p}<0.0001$, reflecting a reliable decrease from sample 2 to sample 3 across groups, $\mathrm{t}(44)=4.639, \mathrm{p}<0.0001$. For plasma, there was also no significant interaction between group and time, $\mathrm{F}(1,46)=0.5877, \mathrm{p}=0.4472$, but a significant effect of time, $\mathrm{F}(1,46)=9.1133$, $p=0.0041$, reflecting a reliable increase from sample 2 to sample 3 across groups, $t(46)=-$ $3.019, \mathrm{p}<0.0041$. Thus, in contradiction of the hypothesis, BDNF concentrations decreased in serum and increased in plasma during rest, independent of the prior intervention. The persistence of the changes BDNF concentrations are visualized for all intervention groups in Figure 3.

Moderate intensity physical exercise 2-3 times per week over 12 weeks improves physical fitness in older adult (H7)

95 participants successfully completed the maximal treadmill test at pretest and 94 at posttest. Following exclusions made for factors that may have influenced the measurements (e.g. current or residual infection), 93 participants were left with $\mathrm{VO}_{2}$ max measurements at pretest $(\mathrm{COG}=21, \mathrm{PE}=25, \mathrm{COG}+\mathrm{PE}=23, \mathrm{PE}+\mathrm{COG}=24)$ and 89 at posttest $(\mathrm{COG}=21, \mathrm{PE}=24$, $\mathrm{COG}+\mathrm{PE}=21, \mathrm{PE}+\mathrm{COG}=23$ ). There was no significant interaction between group and time, $\mathrm{F}(1,87.1)=0.47, \mathrm{p}=0.49$, and no main effect of time, $\mathrm{F}(1,86.3)=1.07, \mathrm{p}=0.30$, or group, 
$\mathrm{F}(1,91.7)=2.23, \mathrm{p}=0.14$. Thus, there were no differential improvement in cardiovascular fitness in the different intervention groups, contradicting the hypothesis.

Cognition in older adults benefits more from repeated sessions of working memory training when each training session is combined with physical exercise, irrespective of order, compared to working memory training alone (H3)

For this hypothesis, groups that received both intervention types $(\mathrm{PE}+\mathrm{COG}, \mathrm{COG}+\mathrm{PE})$ were combined into one group (COMB) and compared to the group that received only cognitive training (COG). The hypothesis concerned performance on trained working memory tasks (trained stimuli, untrained stimuli; $\alpha_{\text {corr }}=0.025$ ), untrained working memory tasks (switching, updating; $\alpha_{\text {corr }}=0.025$ ), untrained cognitive domains (episodic memory, processing speed, spatial reasoning, verbal reasoning; $\alpha_{\text {corr }}=0.0125$ ) and training progress (non-adaptive n-back; $\alpha=0.05)$.

No significant interaction was detected between intervention (COMB, COG) was detected for any of the cognitive composites (H3a-c; trained working memory in Figure 4A) nor on training progress (H3d; Figure 4D), providing no support for a hypothesized difference in cognitive outcome between groups that received both cognitive training in combination with physical exercise (S9 for statistics). There was a significant main effect of time (pretest, posttest) for the same cognitive composites as for $\mathrm{H} 1$, reflecting a general improvement from pretest to posttest, as well as a significant main effect of training visit (1-32) on n-back performance, reflecting a linear improvement in performance across the cognitive training intervention.

Cognition in older adults benefits more from repeated sessions of physical exercise when each exercise session is combined with working memory training, irrespective of order, compared to physical exercise alone (H4) 
Groups that received both intervention types $(\mathrm{PE}+\mathrm{COG}, \mathrm{COG}+\mathrm{PE})$ were combined into one group (COMB) and compared to the group that received only physical exercise (PE). H4 concerned the same cognitive outcomes as $\mathrm{H} 1$ and $\mathrm{H} 2$, with the exception of training progress (not available for the PE group).

A significant interaction was detected between intervention (COMB, PE) and time (pretest, posttest) for trained working memory tasks (trained stimuli \& untrained stimuli; Figure 4B), reflecting greater improvements for the group that was exposed to the cognitive training $(\mathrm{COMB})$ relative to the group that was not $(\mathrm{PE})$. There was no statistically significant intervention-by-time interaction for any other cognitive composite, providing no evidence that the cognitive training resulted in improvements beyond the trained tasks (S10 for statistics). There was a significant main effect of time (pretest, posttest) for the same cognitive composites as for $\mathrm{H} 1$ and $\mathrm{H} 3$, reflecting a general improvement in performance at posttest relative to pretest, as well as a significant main effect of training visit (1-32) on n-back performance, reflecting a linear improvement in performance across the cognitive training intervention.

\section{Cognition in older adults benefits more from repeated sessions of working memory training when each training session is directly preceded as opposed to followed by physical exercise (H1)}

To test this hypothesis, analyses were restricted to the groups that received both intervention types but in different order: $\mathrm{PE}+\mathrm{COG}$ and $\mathrm{COG}+\mathrm{PE}$. The hypothesis concerned the same cognitive constructs as $\mathrm{H} 3$. No significant interaction was detected between intervention $(\mathrm{COG}+\mathrm{PE}, \mathrm{PE}+\mathrm{COG})$ and time (pretest, posttest) for any of the cognitive composites (H1a-c; trained working memory in Figure 4C) nor on training progress (H1d; Figure 4E), contradicting the hypothesized difference in cognitive outcome when cognitive training is preceded as opposed to followed by physical exercise (S8 for statistics). There was a 
significant main effect of time (pretest, posttest) for performance on trained working memory tasks (trained stimuli \& untrained stimuli), untrained working memory tasks (updating), and untrained cognitive domains (episodic memory \& spatial reasoning), reflecting improved performance at posttest compared to pretest, as well as a significant main effect of training visit (1-32) on n-back performance, reflecting a linear improvement over the course of the cognitive training intervention. 

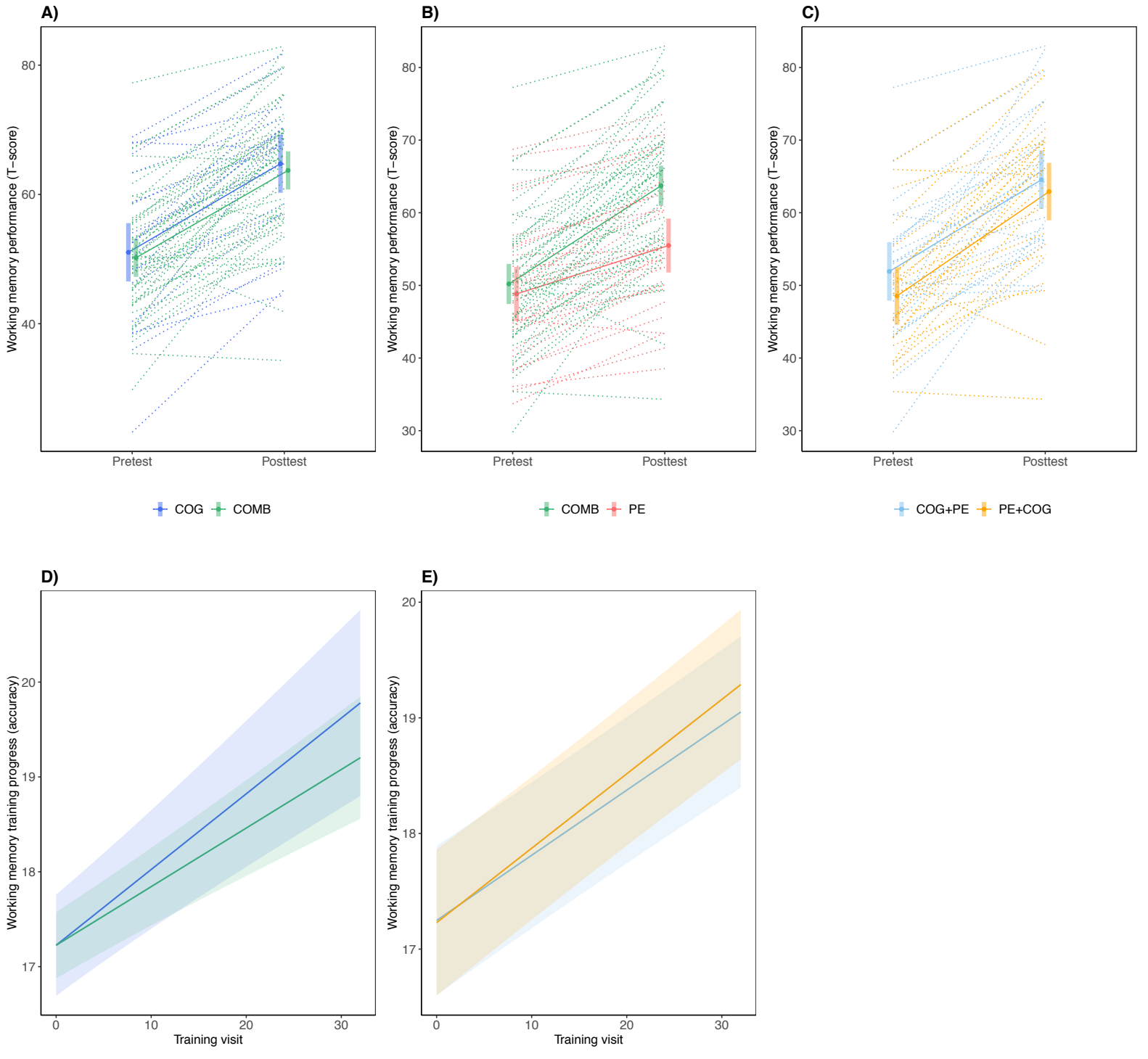

$\boxminus \operatorname{COG} \square$ сOMB

$\square$ COG+PE $\square$ PE+COG

Figure 4. Change in cognitive performance from pretest to posttest (A-C) and across cognitive training visits (DE). Estimated marginal means (error bars $=95 \%$ confidence intervals) derived from linear mixed models testing intervention effects on performance in trained tasks (trained stimuli), in the context of hypothesis 3 (A), hypothesis 4 (B) and hypothesis 1 (C), with spaghetti lines corresponding to individual change trajectories (Tscores, $M=50, S D=10)$. Model-implied regression lines illustrating the effect of training visit on progress in the n-back task during the cognitive training intervention (maximum score $=24$ ), in the context of hypothesis 3 (D) and 4 (E). COG $+\mathrm{PE}=$ cognitive training + physical exercise, $\mathrm{PE}+\mathrm{COG}=$ physical exercise + cognitive training, $\mathrm{COG}=$ cognitive training, $\mathrm{PE}=$ physical exercise, $\mathrm{COMB}=\mathrm{PE}$ and $\mathrm{COG}$, irrespective of order. 
The relationship between change in peripheral BDNF levels in response to physical exercise at pretest and the outcome of repeated sessions of working memory training is greater when each training session is directly preceded as opposed to followed by physical exercise (H2)

To test $\mathrm{H} 2$, analyses were restricted to the groups that received both intervention types but in different order: $\mathrm{PE}+\mathrm{COG}$ and $\mathrm{COG}+\mathrm{PE}$. $\mathrm{H} 2$ concerned the relationship between BDNF concentrations in plasma and in serum, and pre-post change in performance on trained working memory tasks (trained stimuli, untrained stimuli; H2a; $\alpha_{\text {corr }}=0.0125$ ), untrained working memory tasks (switching, updating; $\mathrm{H} 2 \mathrm{~b} ; \alpha_{\text {corr }}=0.0125$ ), untrained cognitive domains (episodic memory, processing speed, spatial reasoning, verbal reasoning; $\mathrm{H} 2 \mathrm{c}$;

$\alpha_{\text {corr }}=0.000625$ ) and training progress (non-adaptive n-back; H2d; $\alpha=0.025$ ). The BDNF measure was the outcome and reflected acute changes in BDNF concentrations following physical exercise at pretest (i.e. sample 2 - sample 1 for $\mathrm{PE}+\mathrm{COG}$ and sample 3 - sample 2 for $\mathrm{COG}+\mathrm{PE}$ ). Considering the high numeric values of the serum concentrations, these were divided by 1000 to achieve a scale more comparable to the cognitive measures. In support of the hypothesis, a significant three-way interaction between group, time and BDNF was found in plasma for performance on trained tasks with untrained stimuli (Table 2). No three-way interaction was detected for any of the remaining cognitive composites, in plasma nor in serum, reflecting support for H2a only (trained working memory tasks; Table 2) and not for $\mathrm{H} 2 \mathrm{~b}-\mathrm{c}$ (working memory tasks and untrained cognitive domain; see complete statistics in S11). 
Table 2. Standardized parameter estimates from the linear mixed effects models and F-tests of main and interaction effects (Type III anova). For unstandardized parameter estimates, see S11. P-values that are below the Bonferroni-corrected significance threshold are highlighted in bold $\left(\alpha_{\text {corr }}=0.0125\right)$. Group $=\mathrm{COG}+\mathrm{PE}<$ $\mathrm{PE}+\mathrm{COG} ;$ Time $=$ pretest $<$ posttest BDNF $=$ change in plasma BDNF concentration following $\mathrm{PE}$ at pretest.

\begin{tabular}{|c|c|c|c|c|c|c|}
\hline \multirow{2}{*}{$\begin{array}{l}\text { Factors } \\
\text { Trained tasks (untrained stimuli) } \\
\quad \text { (Intercept) }\end{array}$} & \multirow[t]{2}{*}{ B } & \multicolumn{2}{|c|}{$\mathrm{Cl}(95 \%)$} & \multirow{2}{*}{$d f(n u m) \quad d f(d n)$} & \multirow[t]{2}{*}{$F$} & \multirow[t]{2}{*}{$p$} \\
\hline & & & & & & \\
\hline Group & -0.1662 & -0.4702 & 0.1378 & 145.00 & 1.15 & 0.2896 \\
\hline Time & 0.3454 & 0.2430 & 0.4479 & 145.00 & 43.72 & $<0.0001$ \\
\hline BDNF & 0.3297 & 0.0972 & 0.5622 & 145.00 & 7.73 & 0.0080 \\
\hline Group x Time & -0.0734 & -0.1758 & 0.0290 & 145.00 & 1.97 & 0.1669 \\
\hline Group x BDNF & 0.0634 & -0.2442 & 0.3709 & 145.00 & 0.16 & 0.6882 \\
\hline Time $x$ BDNF & 0.0938 & -0.0102 & 0.1977 & 145.00 & 3.13 & 0.0834 \\
\hline Group x Time x BDNF & 0.1441 & 0.04025 & 0.2480 & 145.00 & 7.39 & 0.0093 \\
\hline \multicolumn{7}{|c|}{$\begin{array}{l}\text { Trained tasks (trained stimuli) } \\
\text { (Intercept) }\end{array}$} \\
\hline Group & -0.0992 & -0.3540 & 0.1558 & 145.00 & 0.58 & 0.4499 \\
\hline Time & 0.5000 & 0.3535 & 0.6459 & 145.00 & 44.88 & $<0.0001$ \\
\hline BDNF & 0.2904 & 0.0954 & 0.4855 & 145.00 & 8.52 & 0.0055 \\
\hline Group x Time & -0.0248 & -0.1710 & 0.1214 & 145.00 & 0.11 & 0.7412 \\
\hline Group x BDNF & -0.0482 & -0.3062 & 0.2100 & 145.00 & 0.13 & 0.7161 \\
\hline Time $x$ BDNF & 0.0944 & -0.0539 & 0.2428 & 145.00 & 1.56 & 0.2187 \\
\hline Group x Time x BDNF & 0.0841 & -0.0643 & 0.2325 & 145.00 & 1.23 & 0.2726 \\
\hline
\end{tabular}

Figure 5 visualizes how the relationship between change in performance from pretest to posttest (training gain) and acute BDNF change following physical exercise at pretest differed in the two groups $(\mathrm{PE}+\mathrm{COG}, \mathrm{COG}+\mathrm{PE})$, consistent with the hypothesized direction of a greater relationship between acute changes in BDNF levels following physical exercise and cognitive training outcome in the $\mathrm{PE}+\mathrm{COG}$ group. Zero-order correlation coefficients differed significantly, which further supports a differential relationship between training gain and BDNF response in the group that received physical exercise before cognitive training compared to the reverse order, Fisher's $Z=2.63, p=0.009$ (two-tailed). Note that the three-way interaction remained significant at the corrected $\alpha$-level even if the two potential outliers in the PE+COG group, visible in Figure 5, were excluded, $F(1,43)=7.20, p=0.010$. The zeroorder correlations also still differed significantly after outlier removal, Fisher's $Z=2.67$, $p=0.008$ (two-tailed). 


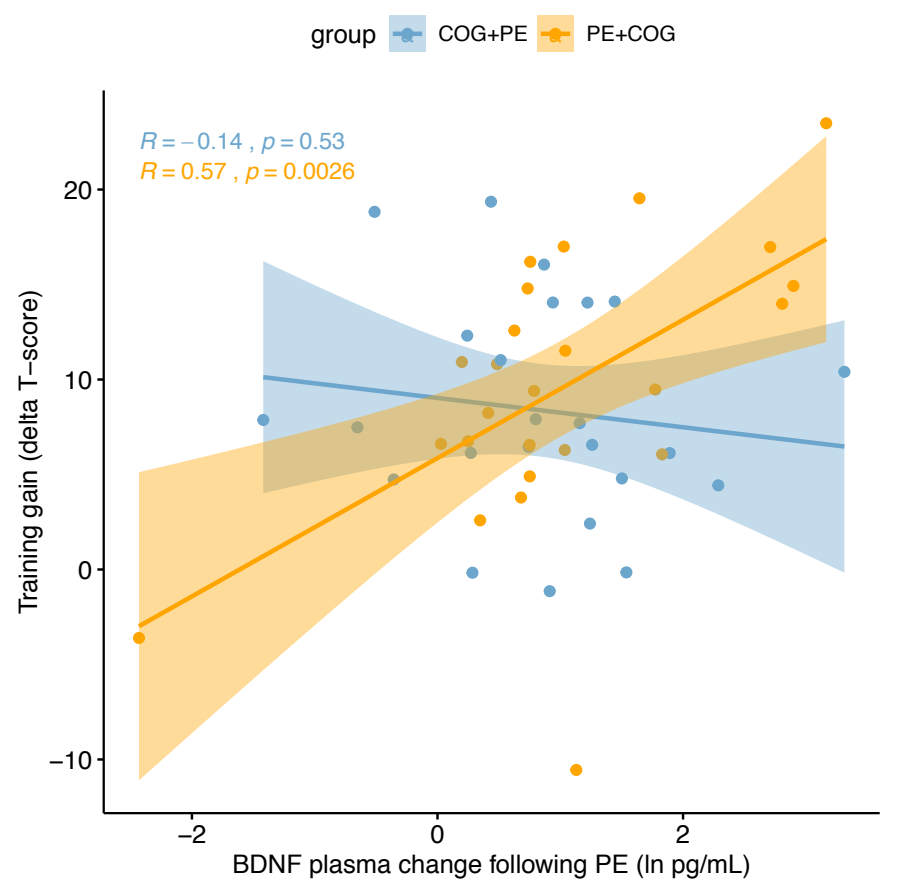

Figure 5. Scatterplot visualizing individual data points and zero-order correlations between training gains (prepost change in performance on trained tasks with untrained stimuli) and acute change in BDNF concentrations in response to physical exercise in plasma at pretest, separate for the group that received physical exercise before cognitive training during the intervention $(\mathrm{PE}+\mathrm{COG})$ and the group that received the same intervention types but in the reverse order (COG+PE). The plot visualizes that a relationship between exercise-induced BDNF change and cognitive training gain only exists in the group that received physical exercise prior to cognitive training. Note that effects remain significant even when the two outliers in the PE $+\mathrm{COG}$ group, both with large negative training gains, are removed.

For cognitive training progress (non-adaptive n-back task; H2d), a trending interaction was detected between time (cognitive training visit 1-32) and acute change in plasma BDNF following PE at pretest, $\mathrm{F}(1,44.15)=5.056, \mathrm{p}=0.029\left(\alpha_{\text {corr }}=0.025\right.$; Figure 6). Inconsistent with the hypothesis of a differential relationship, however, the groups $(\mathrm{COG}+\mathrm{PE}, \mathrm{PE}+\mathrm{COG}) \mathrm{did}$ not differ in regards to the strength of this relationship, $\mathrm{F}(1,44.15)=0.0001, \mathrm{p}=0.99096$ (S11). 


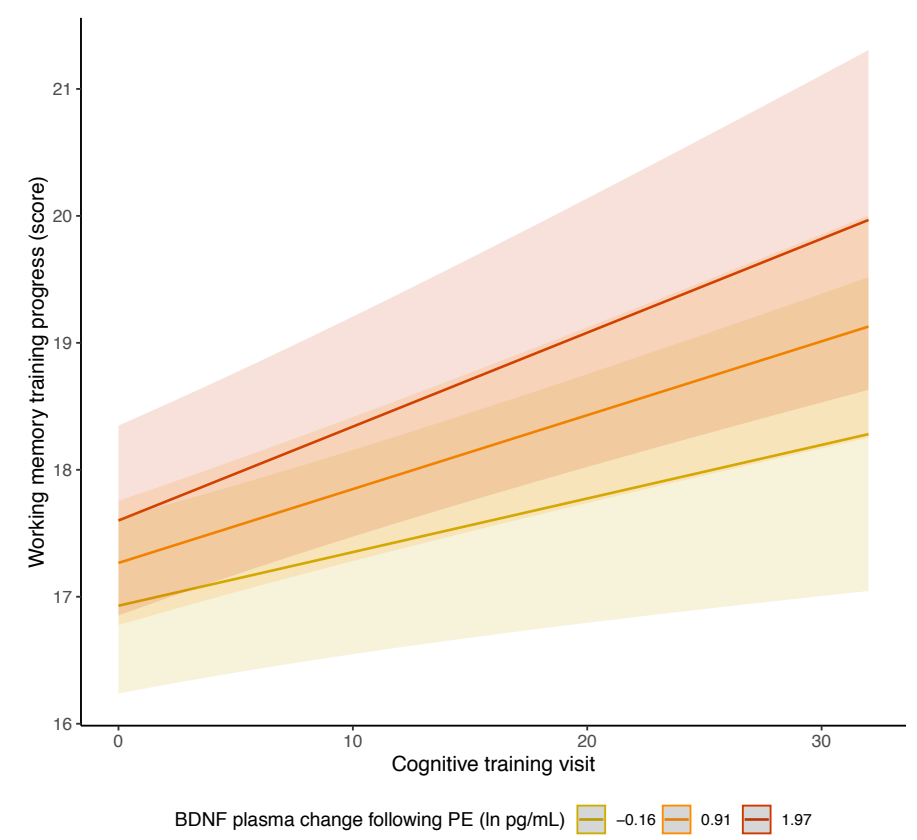

Figure 6. Line plot visualizing the model-implied relationship between acute change in BDNF plasma concentrations following $\mathrm{PE}$ at pretest and linear training progress in a non-adaptive n-back across the cognitive training intervention (visit 1-32). Categorization for change in BDNF concentration has been applied for visualization purposes only and correspond to the average (orange) and one standard deviation below (yellow) and above the average (red).

Significant main effects of change in BDNF plasma concentrations following PE were noted in plasma for performance on trained tasks with trained stimuli as well as with untrained stimuli, reflecting a positive relationship between working memory performance and BDNF response, across time and groups (Table 2). A trending main effect of change in BDNF plasma concentrations was also detected for performance on untrained working memory tasks, $F(1,45.0)=5.45, p=0.024\left(\alpha_{\text {corr }}=0.0125\right)$. No other main effects or two-way interactions with BDNF concentration change following PE were detected for any other cognitive composite, in plasma nor in serum (S11). 


\section{Follow-up analyses}

An important caveat with the demonstrated support for the hypothesis of a differential BDNF

- training gain relationship is that the acute increase in plasma concentrations was not exclusive to physical exercise but occurred also after cognitive training. In a set of exploratory linear mixed models, we therefore examined the three-way interaction effect on BDNF plasma concentrations at the three sampling timepoints at pretest separately: before the first intervention (sample 1), after the first intervention (sample 2) and after the second intervention (sample 3). The interaction was significant for sample 2, $F(1,45.0)=13.276$, $\mathrm{p}<0.001$, and for sample $3, \mathrm{~F}(1,45.0)=5.564, \mathrm{p}=0.023$, but not for sample $1, \mathrm{~F}(1,45.0)=0.289$, $p=0.594$. As visualized in Figure 7A-C, the zero-order correlation between BDNF plasma concentrations and training gain was only reliable in the $\mathrm{PE}+\mathrm{COG}$ group at sample 2, at which point the correlation also differed significantly from the COG+PE group, Fisher's $Z=2.58, p=0.001$ (two-tailed). The zero-order correlations were not significant in either of the groups at sample 1 and 3, although correlation coefficients differed significantly at sample 3, Fisher's $Z=2.21, p=0.027$, but not at sample 1, Fisher's $Z=0.42, p=0.675$ (two-tailed). Additionally, in the groups that received only physical exercise or only cognitive training during the intervention, no significant correlations were detected between pre-post change in performance on trained tasks and BDNF plasma concentrations at any of the sampling timepoints (S12). Thus, BDNF plasma concentrations only appear to predict cognitive training gains when measured immediately after physical exercise and just before cognitive training. 

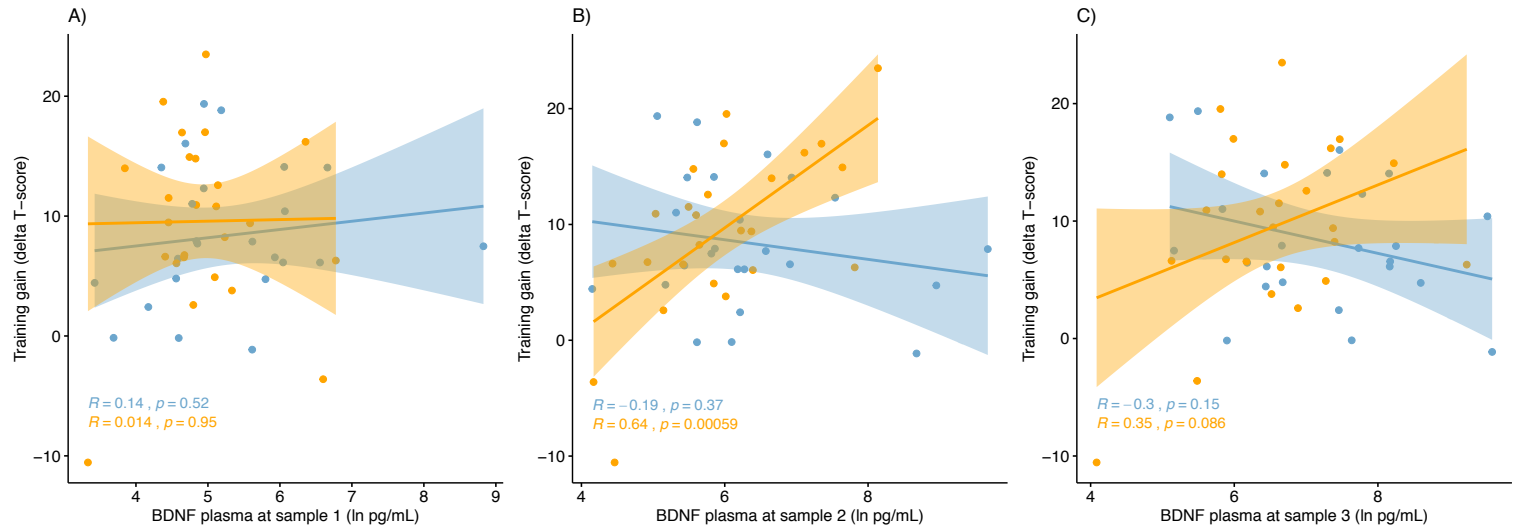

Figure 7. Scatterplots visualizing individual data points and zero-order correlations between training gains (prepost change in performance on trained tasks with untrained stimuli) and BDNF concentrations in sample 1 (A), sample 2 (B) and sample $3(\mathrm{C})$ at pretest, separate for the group that received physical exercise before cognitive training during the intervention ( $\mathrm{PE}+\mathrm{COG}$ : orange) and the group that received the same intervention types but in the reverse order (COG+PE: pale blue). BDNF concentrations only predict cognitive training gain in the $\mathrm{PE}+\mathrm{COG}$ group and only at Sample 2 .

Another important consideration is that the demonstrated support for the hypothesis was limited to the cognitive composite that captured pre-post change in performance on trained working memory tasks with untrained stimuli. No corresponding evidence for a differential association in the $\mathrm{PE}+\mathrm{COG}$ and $\mathrm{COG}+\mathrm{PE}$ groups between BDNF plasma concentrations and training gains was found for performance on trained working memory tasks with trained stimuli. This pattern of findings was not due to differential internal consistency reliability for the two composites, as indicated by equivalent split-half correlations (calculated between odd and even runs/trials at pretest, with Spearman-Brown adjustment) for the composite for trained stimuli $\left(r_{\mathrm{adj}}=0.94\right)$ and for untrained stimuli $\left(r_{\mathrm{adj}}=0.92\right)$.

To explore the differential effects for the two trained composites further, the influence of BDNF plasma concentrations at the three sampling timepoints on training gains were evaluated for each individual test. The three-way interaction, between group $(\mathrm{COG}+\mathrm{PE}<\mathrm{PE}+\mathrm{COG})$, time (pretest $<$ posttest) and BDNF (continuous), was again present at 
sample 2, for running span with trained stimuli, $F(1,45.0)=6.3771, p=0.0152$, running span with untrained stimuli, $F(1,45.0)=6.8023, p=0.01231$, n-back with untrained stimuli, $F(1,45.0)=5.3366, p=0.02552$, but not for $n$-back with trained stimuli, $F(1,45.0)=0.6342$, $p=0.4300$. When considering a unit-weighted composite of all four trained tests, the three-way interaction was again significant only at sample $2, F(1,45.0)=4.3407, p=0.0429$. Thus, whilst support for a differential BDNF - training gain relationship was only detected for trained tasks with untrained stimuli, post-hoc analyses indicated that similar effects may be at play also for trained tasks with trained stimuli.

In regards to the differential relationship between change in plasma BDNF following PE and cognitive training gain in the two combined intervention groups, it is also important to ask whether the two groups differed in the size of the BDNF change. An exploratory Welch twosample t-test revealed that the plasma BDNF change following exercise was equivalent in the $\mathrm{PE}+\mathrm{COG}(M=1.02 \log \mathrm{pg} / \mathrm{mL}, S D=1.15)$ and $\mathrm{COG}+\mathrm{PE}$ groups $(M=0.83 \log \mathrm{pg} / \mathrm{mL}$, $S D=1.00), t(46.57)=-0.61, \mathrm{p}=0.55$, which contradicts the possibility that such a group difference can explain the differential relationship with training gain.

\section{Discussion}

The present study investigated the effect a 12-week multidomain intervention comprising cognitive training and physical exercise in close temporal succession in older adults, focusing on BDNF as a potential mechanism. Consistent with previous meta-analyses, there was no additive effect of the combined intervention compared to cognitive training alone when the timing of cognitive training and physical exercise was not considered (Gheysen et al., 2018; Zhu, Yin, Lang, He, \& Li, 2016). The primary hypothesis concerned the importance of such timing and stated that cognition would benefit more when each cognitive training session is preceded as opposed to followed by physical exercise, consistent with a transient effect of physical exercise on peripheral BDNF and its presumed neuroplastic consequences. Contrary 
to this hypothesis, however, average cognitive outcome did not differ depending on whether participants received physical exercise before or after cognitive training during the intervention. Critically, this was the case even in the presence of reliable increases in peripheral BDNF following physical exercise at pretest, in serum and in plasma, contradicting any average benefit of increased peripheral BDNF post physical exercise on subsequent cognitive engagement. At an interindividual level, however, greater increases of plasma BDNF following physical exercise were found to be associated with greater cognitive training gains, but only when such increases preceded cognitive training. This provides the first empirical support for a time-critical but advantageous role of increases in peripheral BDNF for cognitive training outcome in older adults. As such, the study has established that postexercise increases in plasma BDNF are related to subsequent learning in older adults, with important implications for the design of future multidomain intervention studies that aim to combat the challenge of late-life cognitive impairment. Whilst this is an important finding, there are several considerations worth making.

Consistent with previous research, plasma concentrations were over 100 times lower than serum concentrations at rest, reflecting that the majority of peripheral BDNF is bound to platelets (Rosenfeld et al., 1995). Resting BDNF concentrations in serum (median=24409 $\mathrm{pg} / \mathrm{mL}$, range $=11598-46013)$ and in plasma (median $=141 \mathrm{pg} / \mathrm{mL}$, range $=28-6820)$ corresponded quite well to previous results in healthy younger adults (20-60 years; $n=140$; median $_{\text {serum }}=22600 \mathrm{pg} / \mathrm{mL}$, range $_{\text {serum }}=1900-51500 ;$ median $_{\text {plasma }}=92.5 \mathrm{pg} / \mathrm{mL}$, range $_{\text {plasma }}=8-$ 927)(Lommatzsch et al., 2005) and in healthy older adults ( $70+$ years; $n=259$; median $\left._{\text {serum }}=21628 \mathrm{pg} / \mathrm{mL}, \mathrm{SD}_{\text {serum }}=10729\right)($ Ziegenhorn et al., 2007). Few studies have investigated the acute effect of physical exercise on peripheral BDNF in older adults and methodological differences make results difficult to compare (Dinoff et al., 2017). Metaanalytic results have indicated that exercise-induced BDNF increases are larger in plasma 
than in serum (Dinoff et al., 2017), with increases in serum ranging from $30 \%$ to $100 \%$ and in plasma from $30 \%$ to $165 \%$ in younger adults (Cho et al., 2012; Ferris, Williams, \& Shen, 2007; Rasmussen et al., 2009; Rojas Vega et al., 2006), with some indication of a faster return to baseline in serum compared to plasma (Gilder, Ramsbottom, Currie, Sheridan, \& Nevill, 2014). In a study similar to the present one, a 17\% serum increase was reported following 35 minutes of moderate aerobic exercise in older adults (Håkansson et al., 2017). In this context, the present findings of a $18 \%$ increase in serum appears relatively consistent with previous research whilst the $300 \%$ increase in plasma must be considered high. Unfortunately, very little is known about exercise-induced increases in plasma and its influences, particularly in older adults, which makes further interpretation of this potential discrepancy difficult (Dinoff et al., 2017; Lommatzsch et al., 2005).

BDNF in serum increased following physical exercise but not following cognitive training, which is consistent with the hypothesis and with previous meta-analytical results (Dinoff et al., 2017). In contrast, plasma BDNF demonstrated a reliable but equivalent increase following both physical exercise and cognitive training. In fact, plasma BDNF increased gradually across the course of the entire session, independent of intervention type. Since the present study did not include a passive control group (no intervention), we can therefore not exclude the possibility that the test situation itself caused the increases of plasma BDNF. It is conceivable that factors common to all interventions, such as the blood sampling procedure or the unfamiliar environment and test procedure, reflected exposure to non-physical stressors. Mechanistic links have indeed been made that BDNF and glucocorticoids work in conjunction in response to acute stress but little is known about the acute effects of non-physical stressors on peripheral BDNF concentrations in humans (Jeanneteau, Garabedian, \& Chao, 2008; Linz et al., 2019). Considering previous demonstrations of decreasing or stable resting BDNF concentrations in plasma over the course of the day, we consider diurnal variation in plasma 
BDNF as a less likely account for the gradual and sizeable increases reported here (Begliuomini et al., 2008; Piccinni et al., 2008).

Importantly, independent of the cause of the increases of plasma BDNF, it was demonstrated that increases that followed physical exercise were only predictive of training gains when cognitive training followed as opposed to preceded physical exercise. This finding is consistent with the proposed importance of timing in order to effectively take advantage of acute peripheral BDNF changes in this kind of multidomain intervention (Walsh et al., 2016) . In a follow-up analysis, plasma BDNF concentrations were considered separately for each of the three blood samples obtained at pretest (at rest, after $1^{\text {st }}$ intervention and after $2^{\text {nd }}$ intervention). It was shown that plasma BDNF concentrations were only predictive of training gains in the second blood sample and only in the group that received physical exercise prior to cognitive training, which is important for two reasons. First, the lack a relationship with plasma BDNF at rest (baseline) in both groups indicates that the large increases brought about by the first intervention, or the test situation, are important for facilitating subsequent learning. Second, the differential association with plasma BDNF after the first intervention supports that the large increases brought about by the first intervention, or the test situation, are only important for training gains if the cognitive engagement immediately follows such increases. Similarly, follow-up analyses in the groups that received only physical exercise or only cognitive training revealed no relationships with plasma BDNF, indicating that the association is indeed specific to the group that received physical exercise prior to cognitive training. Related to the previous discussion on causal inference, however, the beneficial effect of acute increases in plasma BDNF when it precedes cognitive engagement may not be specific to physical exercise but could be relevant for any factor that increases plasma BDNF. In contrast to the demonstrated differential association between plasma BDNF increases and training gains from pretest to posttest by intervention order, no corresponding effect was 
found for the rate of training progress during the 12-week intervention. The reason for this can only be speculated on but considering that the non-adaptive n-back task was administered after each cognitive training session, approximately 35 minutes after cessation of the physical exercise, it is possible that plasma BDNF concentrations were returning towards baseline at this point. The finding that BDNF plasma concentrations predicted training gains when measured immediately after physical exercise but not after 30 minutes of cognitive training is consistent with the possibility that any advantageous effect may have faded by the time the non-adaptive n-back task was completed.

It is important to note that the advantageous effect of increases in plasma BDNF following physical exercise for cognitive training outcome was limited to one of the two cognitive composites capturing performance on the working memory updating tests that were trained in the cognitive training (n-back, running span). Specifically, the effect was limited to the composite that captured performance on trained tests that used stimuli that were not seen during the cognitive intervention. In a post-hoc analysis of the individual tests, however, the effect was not only present in the two trained tests with untrained stimuli but also in one of the trained tests with trained stimuli, providing some indication that the effect may be general to performance on the tests that constituted the cognitive training. The effect was also not found for any of the other cognitive composites, suggesting that any effects on training gains did not generalize to performance in other working memory tasks or to other cognitive domains. As such, any advantageous effect appears to be restricted to the specific cognitive tests that coincides with the post-exercise plasma BDNF increase.

Beyond associations with cognitive training outcome, the results provided further support for a positive role of post-exercise increases in plasma BDNF for cognition in general. Specifically, such increases were associated with greater performance on trained working memory tests (both trained and untrained stimuli), across timepoints and independent of 
intervention order, with a similar trend for performance on working memory updating tests that were not trained during the intervention. A trending association was also found between post-exercise increases of plasma BDNF and faster improvements in the non-adaptive updating task that was administered after each cognitive training session, independent of the order of the interventions. Considering that effects were restricted to training progress and to performance on working memory updating tasks, it can be speculated that individuals who demonstrate large increases in plasma BDNF following physical exercise have an advantage in maintaining and continuously updating information in working memory.

It is worth emphasizing that exercise-induced increases in serum BDNF had no advantageous effect on training gains in any of the groups or on any of the cognitive composites, across timepoints, which question the relevance of serum BDNF for cognition. The sources and mechanisms behind exercise-induced BDNF changes in serum and plasma BDNF are not well understood (Walsh \& Tschakovsky, 2018). However, considering that platelets are capable of taking up BDNF but not of synthesizing it (Fujimura et al., 2002), it has been proposed that plasma concentrations are more likely to reflect BDNF secreted from the brain (Rasmussen et al., 2009). The present results appear consistent with the proposal of plasma BDNF is more relevant for cognitive function than serum BDNF but more research is needed before any conclusions can be made.

Considering the demonstrated importance of interindividual differences in the post-exercise BDNF increase in plasma for cognitive training gains, it may seem surprising that no difference in cognitive training outcome was found between the group that received physical exercise before as opposed to after cognitive training. Here it is important to emphasize that in addition to its effects on neurotrophins, physical exercise gives rise to a range of other neurochemical changes in the acute term, including changes in lactate, cortisol, dopamine, norepinephrine, serotonin, GABA and glutamate (Basso \& Suzuki, 2017). Furthermore, 
factors such as experienced exhaustion, sweating, and general arousal following physical exercise are likely to influence subsequent cognitive performance. Therefore, at the group level, it may be that the any beneficial effect of increased plasma BDNF on subsequent cognition is counteracted by other temporally coupled exercise-induced changes. In other words, whilst a particularly large plasma BDNF increase may allow for a beneficial effect to emerge even in the face of such counteracting effects (that may affect most subjects in a similar way), the group average increase may not be differential because of the counteracting effects. In this context, it is interesting to note that previous research suggests that greater cardiovascular fitness could be associated with greater acute BDNF increases following physical exercise (Dinoff et al., 2017). Whilst the hypotheses considered here were concerned with acute and not chronic effects of physical exercise on BDNF and cognition, it is possible an improvement in cardiovascular fitness following the exercise intervention in the present study would have had a boosting effect on the acute BDNF increase and thereby its advantageous effect on cognition, even in the face of counteracting factors. A more exhaustive investigation of exercise-induced neurochemical and related changes will be necessary to better understand the role of plasma BDNF and its interacting counterparts for subsequent cognitive engagement.

In summary, we provide evidence for a time-critical role of acute changes in peripheral BDNF following physical exercise at an interindividual level, by which individuals with greater increases in plasma BDNF following physical exercise at pretest exhibited greater cognitive training gains, but only if each cognitive training session was preceded as opposed to followed by physical exercise. As such, the results provide important mechanistic insights into how multidomain interventions may be delivered for optimal preventative effects in the future. More research will, however, be required to further elucidate the factors that contribute 
to and interact with acute changes in plasma BDNF and how such changes more precisely benefits cognitive training outcome in older age. 


\section{References}

Basso, J. C., \& Suzuki, W. A. (2017). The Effects of Acute Exercise on Mood, Cognition, Neurophysiology, and Neurochemical Pathways: A Review. Brain Plasticity, 2(2), 127-152. https://doi.org/10.3233/BPL-160040

Bates, D., Maechler, M., Bolker, B., \& Walker, S. (2015). Fitting Linear Mixed-Efffects Models Using \{lme4\}. Journal of Statistical Software, 67(1), 1-48. https://doi.org/10.18637/jss.v067.i01

Begliuomini, S., Lenzi, E., Ninni, F., Casarosa, E., Merlini, S., Pluchino, N., ... Genazzani, A. R. (2008). Plasma brain-derived neurotrophic factor daily variations in men: Correlation with cortisol circadian rhythm. The Journal of Endocrinology, 197(2), 429-435. https://doi.org/10.1677/JOE-07-0376

Binder, D. K., \& Scharfman, H. E. (2004). Brain-derived neurotrophic factor. Growth Factors (Chur, Switzerland), 22(3), 123-131.

Björkman, F., Ekblom-Bak, E., Ekblom, Ö., \& Ekblom, B. (2016). Validity of the revised Ekblom Bak cycle ergometer test in adults. European Journal of Applied Physiology, 116, 1627-1638. https://doi.org/10.1007/s00421-016-3412-0

Cho, H.-C., Kim, J., Kim, S., Son, Y. H., Lee, N., \& Jung, S. H. (2012). The concentrations of serum, plasma and platelet BDNF are all increased by treadmill $\mathrm{VO}_{2}$ max performance in healthy college men. Neuroscience Letters, 519(1), 78-83.

https://doi.org/10.1016/j.neulet.2012.05.025

Colcombe, S., \& Kramer, A. F. (2003). Fitness effects on the cognitive function of older adults: A meta-analytic study. Psychological Science, 14(2), 125-130. https://doi.org/10.1111/1467-9280.t01-1-01430

Daskalopoulou, C., Stubbs, B., Kralj, C., Koukounari, A., Prince, M., \& Prina, A. M. (2017). Physical activity and healthy ageing: A systematic review and meta-analysis of 
longitudinal cohort studies. Ageing Research Reviews, 38, 6-17.

https://doi.org/10.1016/j.arr.2017.06.003

Dinoff, A., Herrmann, N., Swardfager, W., \& Lanctôt, K. L. (2017). The effect of acute exercise on blood concentrations of brain-derived neurotrophic factor in healthy adults: A meta-analysis. European Journal of Neuroscience, 46(1), 1635-1646. https://doi.org/10.1111/ejn.13603

Ekblom-Bak, E., Björkman, F., Hellenius, M.-L., \& Ekblom, B. (2014). A new submaximal cycle ergometer test for prediction of VO2max. Scandinavian Journal of Medicine \& Science in Sports, 24(2), 319-326. https://doi.org/10.1111/sms.12014

Erickson, K. I., Prakash, R. S., Voss, M. W., Chaddock, L., Heo, S., McLaren, M., ... Kramer, A. F. (2010). Brain-Derived Neurotrophic Factor Is Associated with AgeRelated Decline in Hippocampal Volume. Journal of Neuroscience, 30(15), 53685375. https://doi.org/10.1523/JNEUROSCI.6251-09.2010

Ferris, L. T., Williams, J. S., \& Shen, C.-L. (2007). The effect of acute exercise on serum brain-derived neurotrophic factor levels and cognitive function. Medicine and Science in Sports and Exercise, 39(4), 728-734. https://doi.org/10.1249/mss.0b013e31802f04c7

Finkel, D., Andel, R., Gatz, M., \& Pedersen, N. L. (2009). The role of occupational complexity in trajectories of cognitive aging before and after retirement. Psychology and Aging, 24(3), 563-573. https://doi.org/10.1037/a0015511

Fujimura, H., Altar, C. A., Chen, R., Nakamura, T., Nakahashi, T., Kambayashi, J., ... Tandon, N. N. (2002). Brain-derived neurotrophic factor is stored in human platelets and released by agonist stimulation. Thrombosis and Haemostasis, 87(4), 728-734.

Gheysen, F., Poppe, L., DeSmet, A., Swinnen, S., Cardon, G., De Bourdeaudhuij, I., ... Fias, W. (2018). Physical activity to improve cognition in older adults: Can physical 
activity programs enriched with cognitive challenges enhance the effects? A systematic review and meta-analysis. International Journal of Behavioral Nutrition and Physical Activity, 15(1), 63. https://doi.org/10.1186/s12966-018-0697-x

Gilder, M., Ramsbottom, R., Currie, J., Sheridan, B., \& Nevill, A. M. (2014). Effect of fat free mass on serum and plasma BDNF concentrations during exercise and recovery in healthy young men. Neuroscience Letters, 560, 137-141. https://doi.org/10.1016/j.neulet.2013.12.034

Håkansson, K., Ledreux, A., Daffner, K., Terjestam, Y., Bergman, P., Carlsson, R., ... Mohammed, A. K. H. (2017). BDNF Responses in Healthy Older Persons to 35 Minutes of Physical Exercise, Cognitive Training, and Mindfulness: Associations with Working Memory Function. Journal of Alzheimer's Disease : JAD, 55(2), 645-657. https://doi.org/10.3233/JAD-160593

Hötting, K., \& Röder, B. (2013). Beneficial effects of physical exercise on neuroplasticity and cognition. Neuroscience and Biobehavioral Reviews, 37(9 Pt B), 2243-2257. https://doi.org/10.1016/j.neubiorev.2013.04.005

Jeanneteau, F., Garabedian, M. J., \& Chao, M. V. (2008). Activation of Trk neurotrophin receptors by glucocorticoids provides a neuroprotective effect. Proceedings of the National Academy of Sciences of the United States of America, 105(12), 4862-4867. https://doi.org/10.1073/pnas.0709102105

Karbach, J., \& Verhaeghen, P. (2014). Making Working Memory Work: A Meta-Analysis of Executive-Control and Working Memory Training in Older Adults. Psychological Science, 25(11), 2027-2037. https://doi.org/10.1177/0956797614548725

Karege, F., Schwald, M., \& Cisse, M. (2002). Postnatal developmental profile of brainderived neurotrophic factor in rat brain and platelets. Neuroscience Letters, 328(3), 261-264. https://doi.org/10.1016/S0304-3940(02)00529-3 
Kelly, M. E., Loughrey, D., Lawlor, B. A., Robertson, I. H., Walsh, C., \& Brennan, S. (2014). The impact of exercise on the cognitive functioning of healthy older adults: A systematic review and meta-analysis. Ageing Research Reviews, 16, 12-31. https://doi.org/10.1016/j.arr.2014.05.002

Kempermann, G., Fabel, K., Ehninger, D., Babu, H., Leal-Galicia, P., Garthe, A., \& Wolf, S. A. (2010). Why and How Physical Activity Promotes Experience-Induced Brain Plasticity. Frontiers in Neuroscience, 4. https://doi.org/10.3389/fnins.2010.00189

Klein, A. B., Williamson, R., Santini, M. A., Clemmensen, C., Ettrup, A., Rios, M., ... Aznar, S. (2011). Blood BDNF concentrations reflect brain-tissue BDNF levels across species. International Journal of Neuropsychopharmacology, 14(3), 347-353. https://doi.org/10.1017/S1461145710000738

Knaepen, K., Goekint, M., Heyman, E. M., \& Meeusen, R. (2010). Neuroplasticity - exerciseinduced response of peripheral brain-derived neurotrophic factor: A systematic review of experimental studies in human subjects. Sports Medicine (Auckland, N.Z.), 40(9), 765-801. https://doi.org/10.2165/11534530-000000000-00000

Kuznetsova, A., Brockhoff, P. B., \& Christensen, R. H. B. (2017). lmerTest Package: Tests in Linear Mixed Effects Models. Journal of Statistical Software, 82(13), 1-26. https://doi.org/10.18637/jss.v082.i13

Lampit, A., Hallock, H., \& Valenzuela, M. (2014). Computerized cognitive training in cognitively healthy older adults: A systematic review and meta-analysis of effect modifiers. PLoS Medicine, 11(11), e1001756. https://doi.org/10.1371/journal.pmed.1001756

Linz, R., Puhlmann, L. M. C., Apostolakou, F., Mantzou, E., Papassotiriou, I., Chrousos, G. P., ... Singer, T. (2019). Acute psychosocial stress increases serum BDNF levels: An 
antagonistic relation to cortisol but no group differences after mental training. Neuropsychopharmacology. https://doi.org/10.1038/s41386-019-0391-y

Lommatzsch, M., Zingler, D., Schuhbaeck, K., Schloetcke, K., Zingler, C., Schuff-Werner, P., \& Virchow, J. C. (2005). The impact of age, weight and gender on BDNF levels in human platelets and plasma. Neurobiology of Aging, 26(1), 115-123. https://doi.org/10.1016/j.neurobiolaging.2004.03.002

Ngandu, T., Lehtisalo, J., Solomon, A., Levälahti, E., Ahtiluoto, S., Antikainen, R., ... Kivipelto, M. (2015). A 2 year multidomain intervention of diet, exercise, cognitive training, and vascular risk monitoring versus control to prevent cognitive decline in atrisk elderly people (FINGER): A randomised controlled trial. The Lancet, 385(9984), 2255-2263. https://doi.org/10.1016/S0140-6736(15)60461-5

Pan, W., Banks, W. A., Fasold, M. B., Bluth, J., \& Kastin, A. J. (1998). Transport of brainderived neurotrophic factor across the blood-brain barrier. Neuropharmacology, 37(12), 1553-1561. https://doi.org/10.1016/S0028-3908(98)00141-5

Piccinni, A., Marazziti, D., Catena, M., Domenici, L., Del Debbio, A., Bianchi, C., ... Dell'Osso, L. (2008). Plasma and serum brain-derived neurotrophic factor (BDNF) in depressed patients during 1 year of antidepressant treatments. Journal of Affective Disorders, 105(1-3), 279-283. https://doi.org/10.1016/j.jad.2007.05.005

Poo, M. M. (2001). Neurotrophins as synaptic modulators. Nature Reviews. Neuroscience, 2(1), 24-32. https://doi.org/10.1038/35049004

R Core Team. (2017). R: A language and environment for statistical computing. $R$ Foundation for Statistical Computing.

Rasmussen, P., Brassard, P., Adser, H., Pedersen, M. V., Leick, L., Hart, E., ... Pilegaard, H. (2009). Evidence for a release of brain-derived neurotrophic factor from the brain 
during exercise. Experimental Physiology, 94(10), 1062-1069.

https://doi.org/10.1113/expphysiol.2009.048512

Ritchie, S. J., \& Tucker-Drob, E. M. (2018). How Much Does Education Improve Intelligence? A Meta-Analysis. Psychological Science, 29(8), 1358-1369. https://doi.org/10.1177/0956797618774253

Rojas Vega, S., Strüder, H. K., Vera Wahrmann, B., Schmidt, A., Bloch, W., \& Hollmann, W. (2006). Acute BDNF and cortisol response to low intensity exercise and following ramp incremental exercise to exhaustion in humans. Brain Research, 1121(1), 59-65. https://doi.org/10.1016/j.brainres.2006.08.105

Rosenfeld, R. D., Zeni, L., Haniu, N., Talvenheimo, J., Radka, S. F., Bennett, L., ... Welcher, A. A. (1995). Purification and Identification of Brain-Derived Neurotrophic Factor from Human Serum. Protein Expression and Purification, 6(4), 465-471. https://doi.org/10.1006/prep.1995.1062

Sasaki, J. E., John, D., \& Freedson, P. S. (2011). Validation and comparison of ActiGraph activity monitors. Journal of Science and Medicine in Sport, 14(5), 411-416. https://doi.org/10.1016/j.jsams.2011.04.003

Smith, P. J., Blumenthal, J. A., Hoffman, B. M., Cooper, H., Strauman, T. A., Welsh-Bohmer, K., ... Sherwood, A. (2010). Aerobic exercise and neurocognitive performance: A meta-analytic review of randomized controlled trials. Psychosomatic Medicine, 72(3), 239-252. https://doi.org/10.1097/PSY.0b013e3181d14633

Soveri, A., Antfolk, J., Karlsson, L., Salo, B., \& Laine, M. (2017). Working memory training revisited: A multi-level meta-analysis of n-back training studies. Psychonomic Bulletin \& Review, 24(4), 1077-1096. https://doi.org/10.3758/s13423-016-1217-0 
Szuhany, K. L., Bugatti, M., \& Otto, M. W. (2015). A meta-analytic review of the effects of exercise on brain-derived neurotrophic factor. Journal of Psychiatric Research, 60, 56-64. https://doi.org/10.1016/j.jpsychires.2014.10.003

Trost, S. G., McIver, K. L., \& Pate, R. R. (2005). Conducting accelerometer-based activity assessments in field-based research. Medicine and Science in Sports and Exercise, 37(11 Suppl), S531-543.

Walsh, J. J., Scribbans, T. D., Bentley, R. F., Kellawan, J. M., Gurd, B., \& Tschakovsky, M. E. (2016). Neurotrophic growth factor responses to lower body resistance training in older adults. Applied Physiology, Nutrition, and Metabolism, 41(3), 315-323. https://doi.org/10.1139/apnm-2015-0410

Walsh, J. J., \& Tschakovsky, M. E. (2018). Exercise and circulating BDNF: Mechanisms of release and implications for the design of exercise interventions. Applied Physiology, Nutrition, and Metabolism, 43(11), 1095-1104. https://doi.org/10.1139/apnm-20180192

WHO. (2012). Dementia: A public health priority. Retrieved from http://www. who.int/mental_health/publications/dementia_report_2012/en

Zhu, X., Yin, S., Lang, M., He, R., \& Li, J. (2016). The more the better? A meta-analysis on effects of combined cognitive and physical intervention on cognition in healthy older adults. Ageing Research Reviews, 31, 67-79. https://doi.org/10.1016/j.arr.2016.07.003

Ziegenhorn, A. A., Schulte-Herbrüggen, O., Danker-Hopfe, H., Malbranc, M., Hartung, H.D., Anders, D., ... Hellweg, R. (2007). Serum neurotrophins-A study on the time course and influencing factors in a large old age sample. Neurobiology of Aging, 28(9), 1436-1445. https://doi.org/10.1016/j.neurobiolaging.2006.06.011 
